\title{
New Findings by High-Order DNS for Late Flow Transition in a Boundary Layer
}

\author{
Chaoqun Liu, ${ }^{1}$ Lin Chen, ${ }^{1,2}$ and Ping Lu ${ }^{1}$ \\ ${ }^{1}$ Mathematics Department, University of TX at Arlington, Arlington, TX 76019, USA \\ ${ }^{2}$ Aerodynamics Department, Nanjing University of Aeronautics and Astronautics, Nanjing 210016, China
}

Correspondence should be addressed to Chaoqun Liu, cliu@omega.uta.edu

Received 6 October 2010; Accepted 24 November 2010

Academic Editor: Guan Yeoh

Copyright ( $) 2011$ Chaoqun Liu et al. This is an open access article distributed under the Creative Commons Attribution License, which permits unrestricted use, distribution, and reproduction in any medium, provided the original work is properly cited.

\begin{abstract}
This paper serves as a summary of new discoveries by DNS for late stages of flow transition in a boundary layer. The widely spread concept "vortex breakdown" is found theoretically impossible and never happened in practice. The ring-like vortex is found the only form existing inside the flow field. The ring-like vortex formation is the result of the interaction between two pairs of counterrotating primary and secondary streamwise vortices. Following the first Helmholtz vortex conservation law, the primary vortex tube rolls up and is stretched due to the velocity gradient. In order to maintain vorticity conservation, a bridge must be formed to link two $\Lambda$-vortex legs. The bridge finally develops as a new ring. This process keeps going on to form a multiple ring structure. The U-shaped vortices are not new but existing coherent vortex structure. Actually, the U-shaped vortex, which is a third level vortex, serves as a second neck to supply vorticity to the multiple rings. The small vortices can be found on the bottom of the boundary layer near the wall surface. It is believed that the small vortices, and thus turbulence, are generated by the interaction of positive spikes and other higher level vortices with the solid wall. The mechanism of formation of secondary vortex, second sweep, positive spike, high shear distribution, downdraft and updraft motion, and multiple ring-circle overlapping is also investigated.
\end{abstract}

\section{Introduction}

The transition process from laminar to turbulent flow in boundary layers is a basic scientific problem in modern fluid mechanics and has been the subject of study for over a century. Many different concepts for the explanation of the mechanisms involved have been developed based on numerous experimental, theoretical, and numerical investigations. After over a century study on the turbulence, the linear and early weakly nonlinear stages of flow transition are pretty well understood. However, for late non-linear transition stages, there are still many questions remaining for research [1-5].

Ring-like vortices play a key role in the flow transition. They generate rapid downward jets (second sweep) which induce the positive spike and bring the high energy to the boundary layer and work together with the upward jets (ejection) to mix the boundary layer. In other words, the ring-like vortex formation and development are a key topic for late flow transition in boundary layer. It appears that there is no turbulence without ring-like vortices. It is natural to give comprehensive study on the ring formation and development.

1.1. Ring-Like Vortex Formation. Hama and Nutant [6] described the process of formation of " $\Omega$-shaped vortex" in the vicinity of the $\Lambda$-vortex tip. They found that "...a simplified numerical analysis indicates that the hyperbolic vortex filament deforms by its own induction into a milk-bottle shape (the " $\Omega$-vortex") and lifts up its tip...". Later, Moin et al. [7] used numerical method with the Biot-Savart law and concluded that the ring is formed through a mechanism of self-induction and the multiple ring formation is a result of first ring "pinching off." They believe that the solution of Navier-Stokes equation does not change the mechanism but may delay the ring "pinching off". However, they may be somewhat right, but what they discussed is not relevant to the boundary layer transition since the ring-like vortex is formed in a boundary layer and viscosity and full 3D time-dependent 


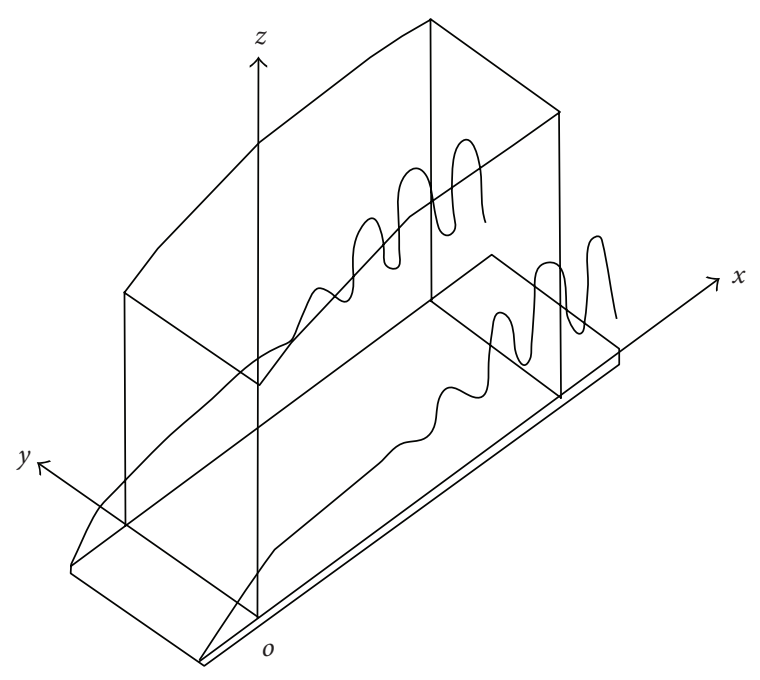

FIgUre 1: Computation domain.

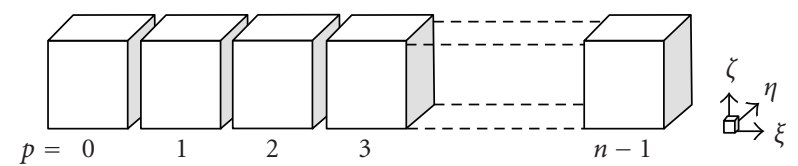

Figure 2: The domain decomposition along streamwise direction in the computational space.

Navier-Stokes equations must be considered. In addition, our new DNS results show, the ring is a perfect circle not a deformed shape, the ring stands perpendicularly with 90 degree not 45 degree, and, more important, "ring pinch off." is never found. The ring-like vortex has its own coherent structure as we show below, which is quite different from what Moin et al. have shown [7].

1.2. Multiple Ring Formation. Crow theory [8] has been considered as the mechanism of multiple ring formation [2]. They pointed out that "the formation of a set of vortex rings from two counter-rotating vortices is usually called "Crow instability." This instability could be involved at the stage of formation of the ring-like vortices due to interaction of the $\Lambda$-vortex legs." However, there is no evidence to prove such a mechanism. We cannot find any links between Crow theory and multiple ring formation. Apparently, Crow theory is not related to multiple ring formation in a boundary layer.

1.3. Vortex Breakdown. "Vortex Breakdown" has been widely spread in many research papers and textbooks [5, 9-11]. However, our analysis found that "Vortex Breakdown" is theoretically impossible. In practice, "Vortex Breakdown" can never happen. It is found that "Vortex Breakdown" described by exiting publications $[5,9,10]$ is either misinterpreted by $2 \mathrm{D}$ visualization or by using the pressure center as the vortex center.

1.4. U-Shaped Vortex. U-shaped vortex [9] or barrel-shaped wave [5] is still a mystery and thought as a newly formed

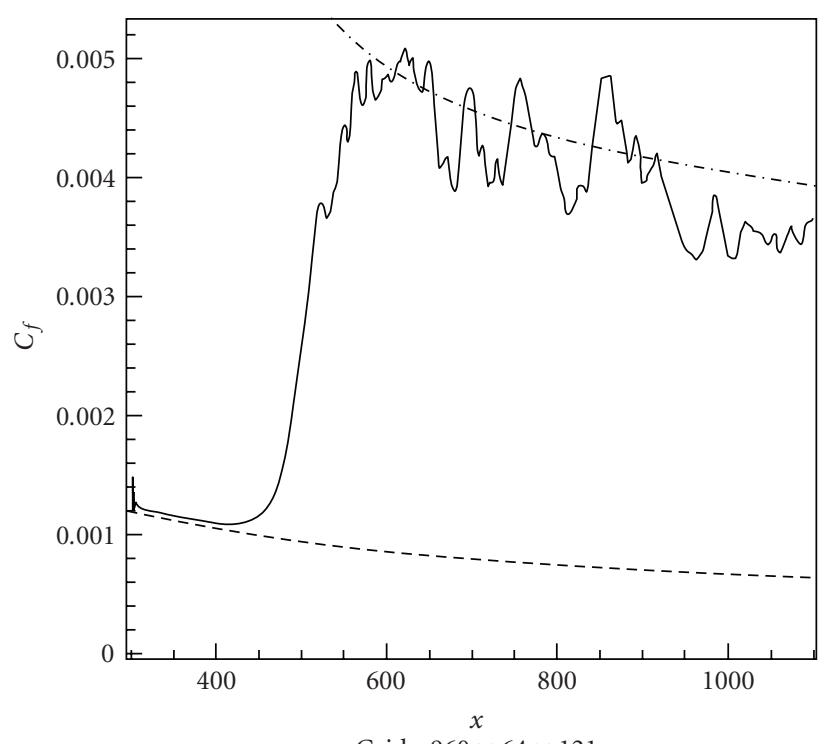

Grids: $960 \times 64 \times 121$

$$
\begin{aligned}
& \text { _ Transitional flow (DNS result) } \\
& - \text { - } \text { Laminar flow } \\
& \text {-.- } \text { Turbulent flow (Cousteix ,1989) }
\end{aligned}
$$

FIGURE 3: Streamwise evolution of the time- and spanwise-averaged skin-friction coefficient.

secondary vortex or waves. However, it is found by our new DNS that the U-shaped vortex was an original coherent structure and it is a tertiary (not secondary) vortex with same vorticity sign as the ring legs. The U-shaped vortices serve as a second neck to supply vorticity to the rings and keep the leading ring alive.

1.5. High Shear Distribution. The high shear (HS) distribution can be found in the paper by Bake et al. [3], but it is questionable why the HS disappears near the neck where the flow is very active. However, our new DNS found that there is no normal direction velocity component and then no sweeps and ejections in that area.

Anyway, there are many questions, which are related to late flow transition, that have not been answered or misinterpreted. In order to get deep understanding on the late flow transition in a boundary layer, we recently conducted a large grid high-order DNS with $1920 \times 241 \times$ 128 gird points and about 600,000 time steps to study the mechanism of the late stages of flow transition in a boundary layer. Many new findings are made, and new mechanisms are revealed. Here, we use the $\lambda_{2}$ criterion [12] for visualization.

The paper is organized in the following way: Section 2 presents the governing Navier-Stokes equations and numerical approaches; Section 3 shows the code verification and validation to make sure that the DNS code is correct; Section 4 provides the new DNS results and summarizes the new discoveries and new mechanism of formation and further development of ring-like vortices. A number of conclusions are made in Section 5. 


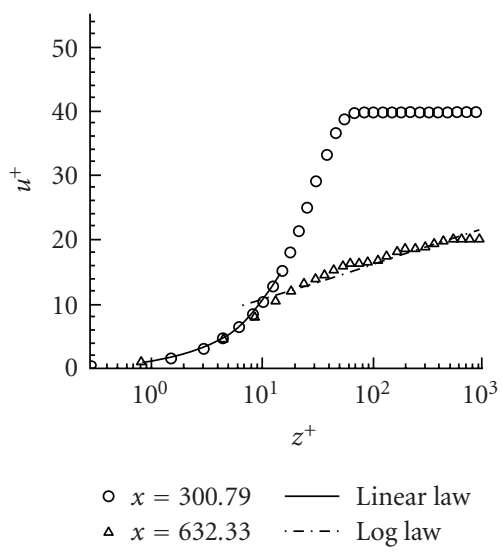

(a) Coarse grids $(960 \times 64 \times 121)$

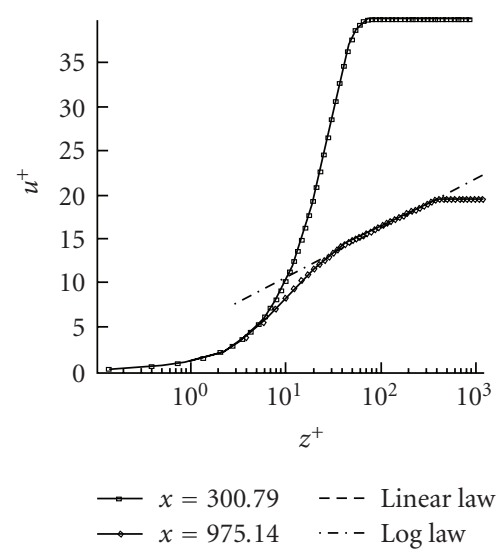

(b) Fine grids $(1920 \times 128 \times 241)$

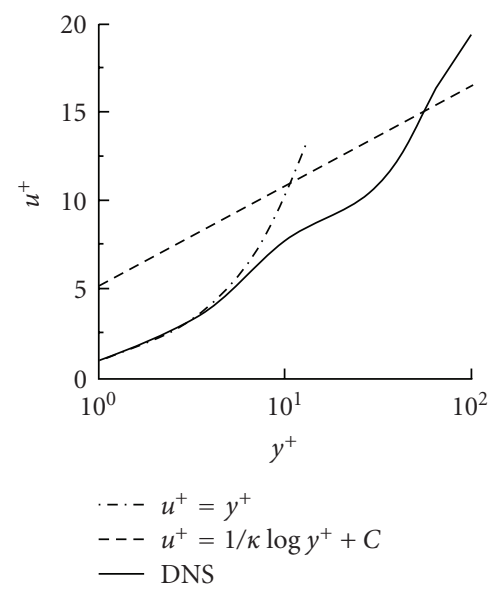

(c) Singer and Joslin [9]

FIGURE 4: Log-linear plots of the time- and spanwise-averaged velocity profile in wall unit.

TABle 1: Flow parameters.

\begin{tabular}{cccccccc}
\hline$M_{\infty}$ & $\mathrm{Re}$ & $x_{\text {in }}$ & $L x$ & $L y$ & $L z_{\text {in }}$ & $T_{w}$ \\
\hline 0.5 & 1000 & $300.79 \delta_{\text {in }}$ & $798.03 \delta_{\text {in }}$ & $22 \delta_{\text {in }}$ & $40 \delta_{\text {in }}$ & $273.15 \mathrm{~K}$ & $273.15 \mathrm{~K}$ \\
\hline
\end{tabular}

\section{Governing Equations and Numerical Methods}

The governing equations we used are the three-dimensional compressible Navier-Stokes equations in generalized curvilinear coordinates and in a conservative form:

$$
\frac{1}{J} \frac{\partial Q}{\partial t}+\frac{\partial\left(E-E_{v}\right)}{\partial \xi}+\frac{\partial\left(F-F_{v}\right)}{\partial \eta}+\frac{\partial\left(H-H_{v}\right)}{\partial \zeta}=0
$$

The vector of conserved quantities $Q$, inviscid flux vector $(E, F, G)$, and viscous flux vector $\left(E_{v}, F_{v}, G_{v}\right)$ are defined as

$$
\begin{gathered}
Q=\left(\begin{array}{c}
\rho \\
\rho u \\
\rho v \\
\rho w \\
e
\end{array}\right), \quad E=\frac{1}{J}\left(\begin{array}{c}
\rho U \\
\rho u U+p \xi_{x} \\
\rho v U+p \xi_{y} \\
\rho w U+p \xi_{z} \\
U(e+p)
\end{array}\right), \\
F=\frac{1}{J}\left(\begin{array}{c}
\rho V \\
\rho v V+p \eta_{x} \\
\rho w V+p \eta_{z} \\
V(e+p)
\end{array}\right), \quad H=\frac{1}{J}\left(\begin{array}{c}
\rho W \\
\rho u W+p \zeta_{x} \\
\rho w W+p \zeta_{y} \\
\rho w \\
W(e+p)
\end{array}\right), \\
E_{v}=\frac{1}{J}\left(\begin{array}{c}
\tau_{x y} \xi_{x}+\tau_{y y} \xi_{y}+\tau_{z y} \xi_{z} \\
\tau_{x z} \xi_{x}+\tau_{y z} \xi_{y}+\tau_{z z} \xi_{z} \\
q_{x} \xi_{x}+q_{y} \xi_{y}+q_{z} \xi_{z}
\end{array}\right),
\end{gathered}
$$

$$
\begin{gathered}
F_{v}=\frac{1}{J}\left(\begin{array}{c}
0 \\
\tau_{x x} \eta_{x}+\tau_{y x} \eta_{y}+\tau_{z x} \eta_{z} \\
\tau_{x y} \eta_{x}+\tau_{y y} \eta_{y}+\tau_{z y} \eta_{z} \\
\tau_{x z} \eta_{x}+\tau_{y z} \eta_{y}+\tau_{z z} \eta_{z} \\
q_{x} \eta_{x}+q_{y} \eta_{y}+q_{z} \eta_{z}
\end{array}\right), \\
H_{v}=\frac{1}{J}\left(\begin{array}{c}
0 \\
\tau_{x x} \zeta_{x}+\tau_{y x} \zeta_{y}+\tau_{z x} \zeta_{z} \\
\tau_{x y} \zeta_{x}+\tau_{y y} \zeta_{y}+\tau_{z y} \zeta_{z} \\
\tau_{x z} \zeta_{x}+\tau_{y z} \zeta_{y}+\tau_{z z} \zeta_{z} \\
q_{x} \zeta_{x}+q_{y} \zeta_{y}+q_{z} \zeta_{z}
\end{array}\right),
\end{gathered}
$$

where $J$ is the Jacobian of the coordinate transformation between the curvilinear $(\xi, \eta, \zeta)$ and Cartesian $(x, y, z)$ frames and $\xi_{x}, \xi_{y}, \xi_{z}, \eta_{x}, \eta_{y}, \eta_{z}, \zeta_{x}, \zeta_{y}, \zeta_{z}$ are coordinate transformation metrics. The contravariant velocity components $U, V, W$ are defined as $U=u \xi_{x}+v \xi_{y}+w \xi_{z}, V=u \eta_{x}+$ $v \eta_{y}+w \eta_{z}, W=u \zeta_{x}+v \zeta_{y}+w \zeta_{z} ; e$ is the total energy. The components of viscous stress and heat flux are denoted by $\tau_{x x}, \tau_{y y}, \tau_{z z}, \tau_{x y}, \tau_{x z}, \tau_{y z}$, and $q_{x}, q_{y}, q_{z}$, respectively.

In the dimensionless form of (1), the reference values for length, density, velocities, temperature, and pressure are $\delta_{\text {in }}, \rho_{\infty}, U_{\infty}, T_{\infty}$, and $\rho_{\infty} U_{\infty}^{2}$, respectively. The Mach number and Reynolds number are expressed as

$$
M_{\infty}=\frac{U_{\infty}}{\sqrt{\gamma R T_{\infty}}}, \quad \operatorname{Re}=\frac{\rho_{\infty} U_{\infty} \delta_{\text {in }}}{\mu_{\infty}},
$$

where $R$ is the ideal gas constant, $\gamma$ the ratio of specific heats, $\delta_{\text {in }}$ the inflow displacement thickness, and $\mu_{\infty}$ the viscosity. 


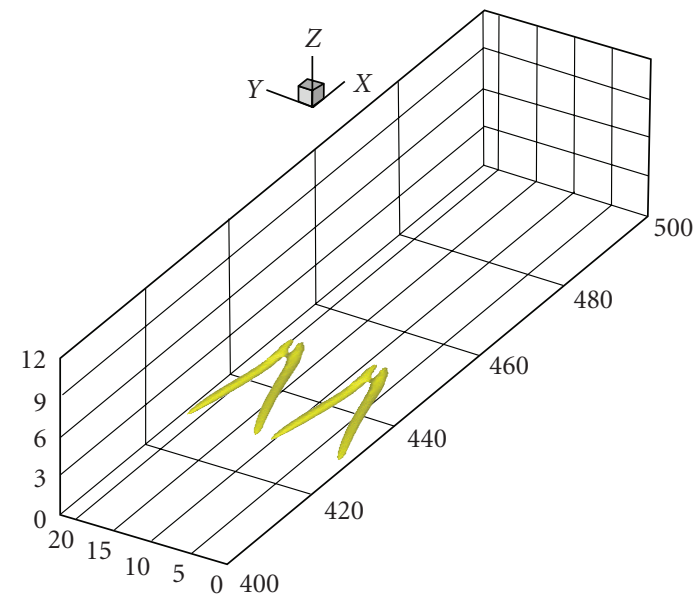

(a) $t=6 \mathrm{~T}$

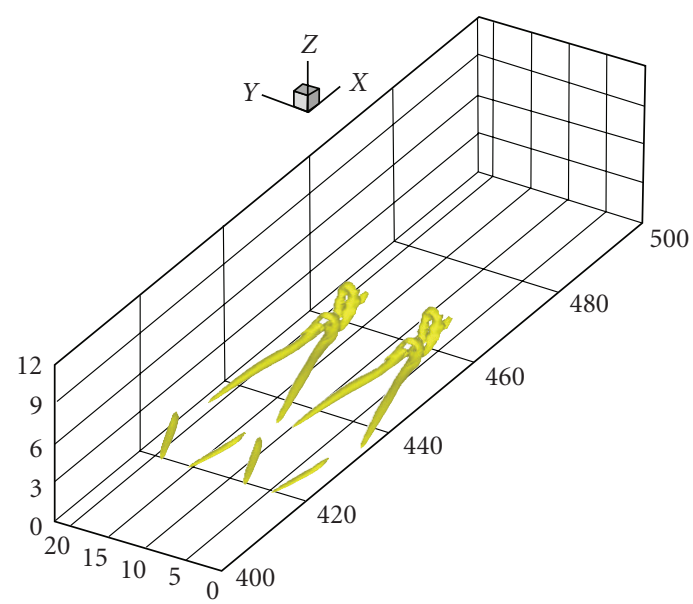

(c) $t=6.4 T$

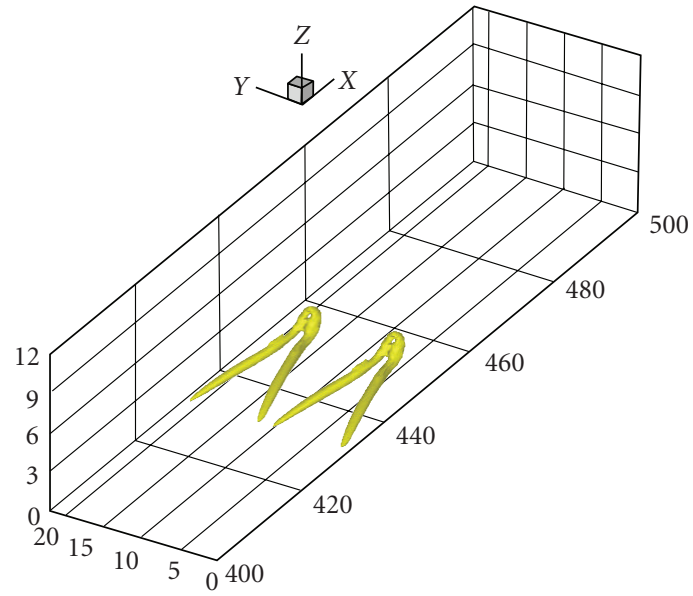

(b) $t=6.2 T$

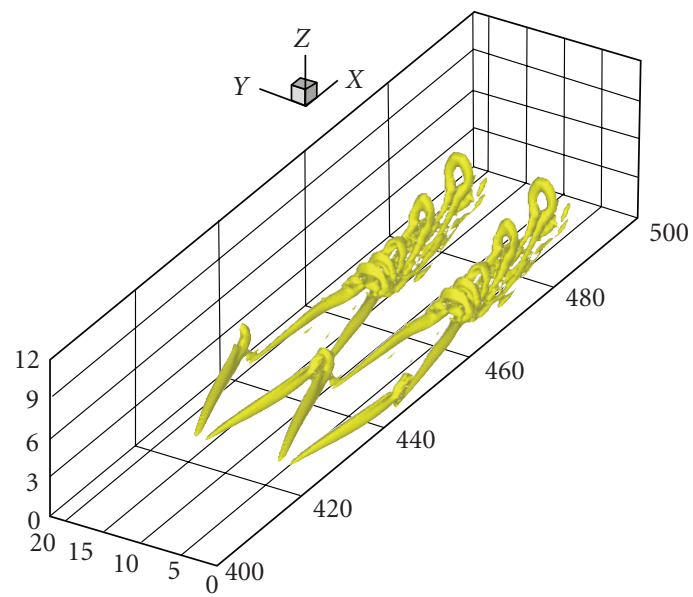

(d) $t=7.0 \mathrm{~T}$

(a)
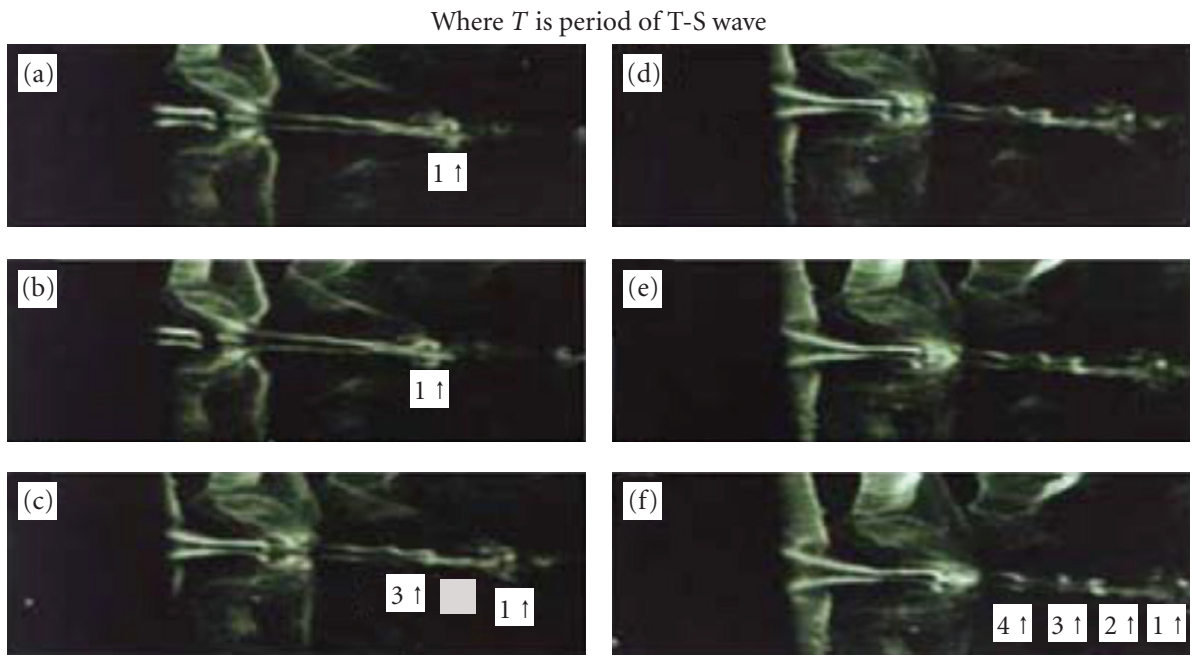

(b)

Figure 5: (A) The evolution of vortex structures at the late stage of transition (where $T$ is period of T-S wave). (B) Evolution of type ring-like vortices chain by experiment [13]. 


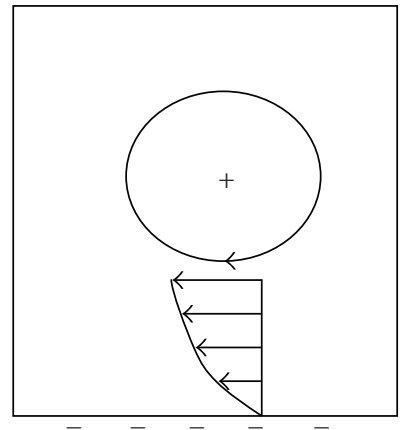

(a)

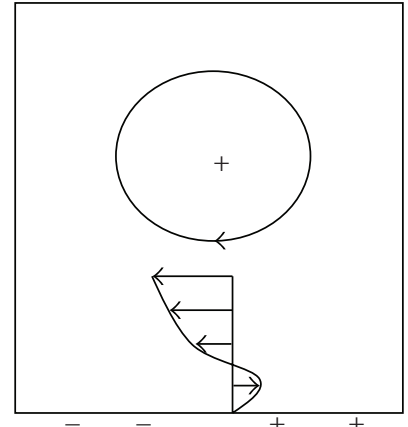

(b)

FIGURE 6: Sketch of secondary vortex and third level vorticity formation.

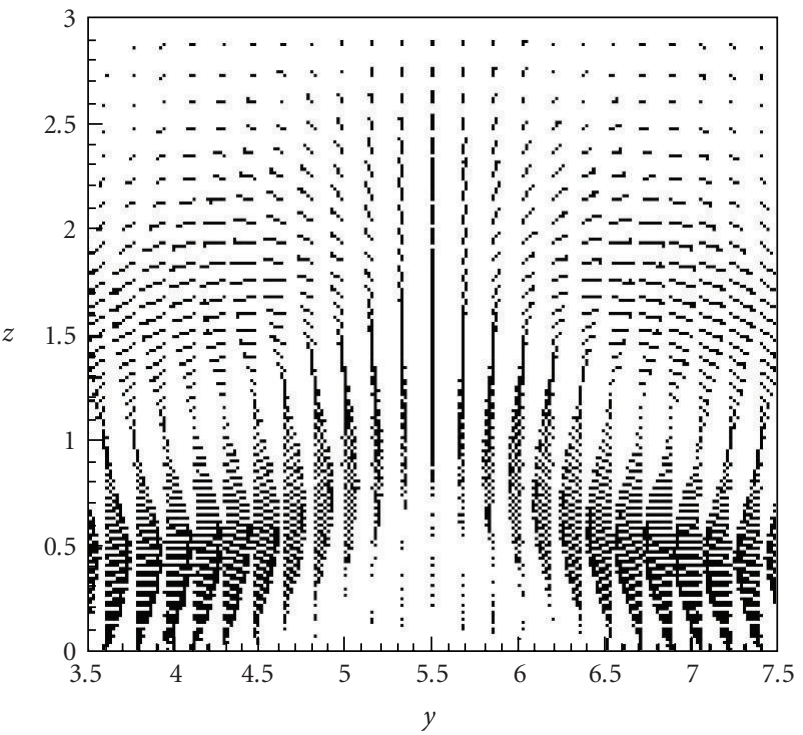

(a) Velocity profile induced by prime vortex

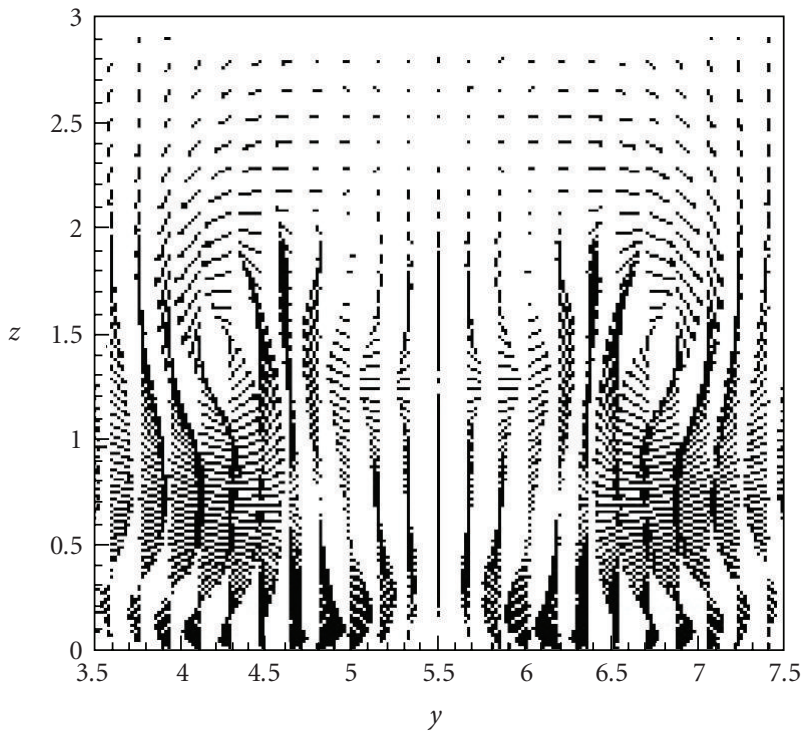

(b) Velocity profile change direction

FIGURE 7: DNS results of secondary vortex and third level vorticity formation.

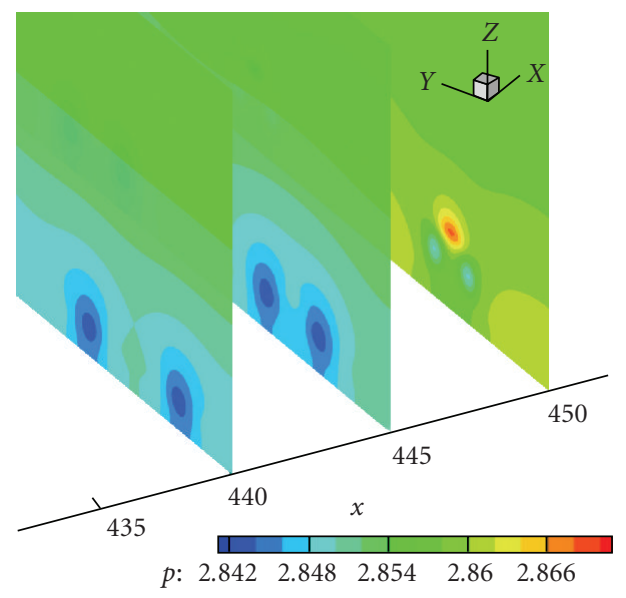

FIgURE 8: Pressure distributions in the evolution of vortex structure $(t=6.2 T)$
A sixth-order compact scheme [14] is used for the spatial discretization in the streamwise and wall normal directions. For internal points $j=3, \ldots, N-2$, the sixth-order compact scheme is as follows:

$$
\begin{aligned}
\frac{1}{3} f_{j-1}^{\prime} & +f_{j}^{\prime}+\frac{1}{3} f_{j+1}^{\prime} \\
& =\frac{1}{h}\left(-\frac{1}{36} f_{j-2}-\frac{7}{9} f_{j-1}+\frac{7}{9} f_{j+1}+\frac{1}{36} f_{j+2}\right),
\end{aligned}
$$

where $f_{j}^{\prime}$ is the derivative at point $j$. The fourth order compact scheme is used at points $j=2, N-1$, and the third order one-sided compact scheme is used at the boundary points $j=1, N$.

In the spanwise direction where periodical conditions are applied, the pseudospectral method is used. In order to eliminate the spurious numerical oscillations caused by central difference schemes, a high-order spatial scheme is 


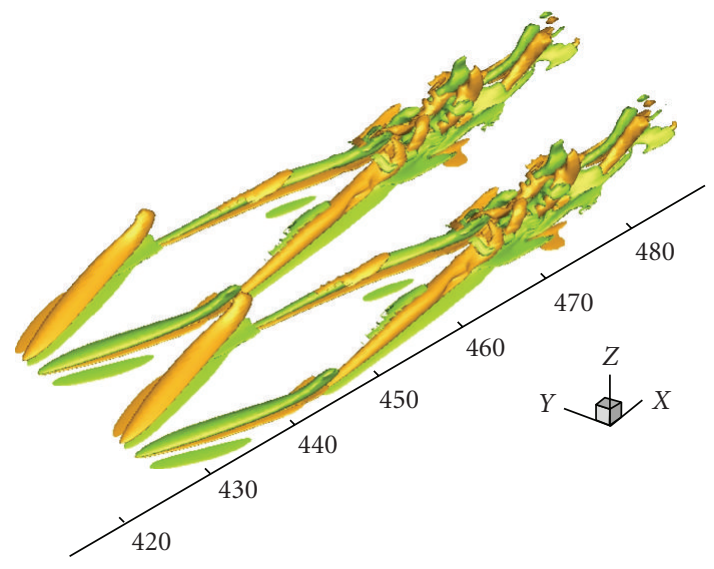

(a)

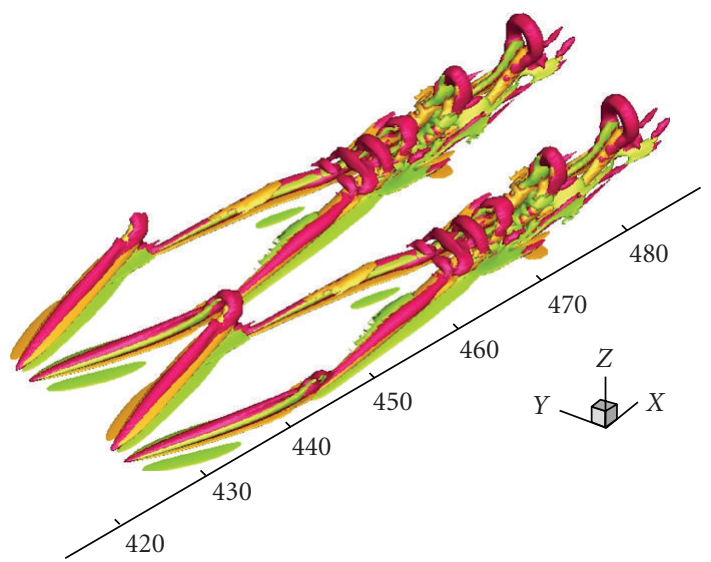

(b)

FIGURE 9: Sketch of the relationship of ring-like vortices and streamwise vortices.

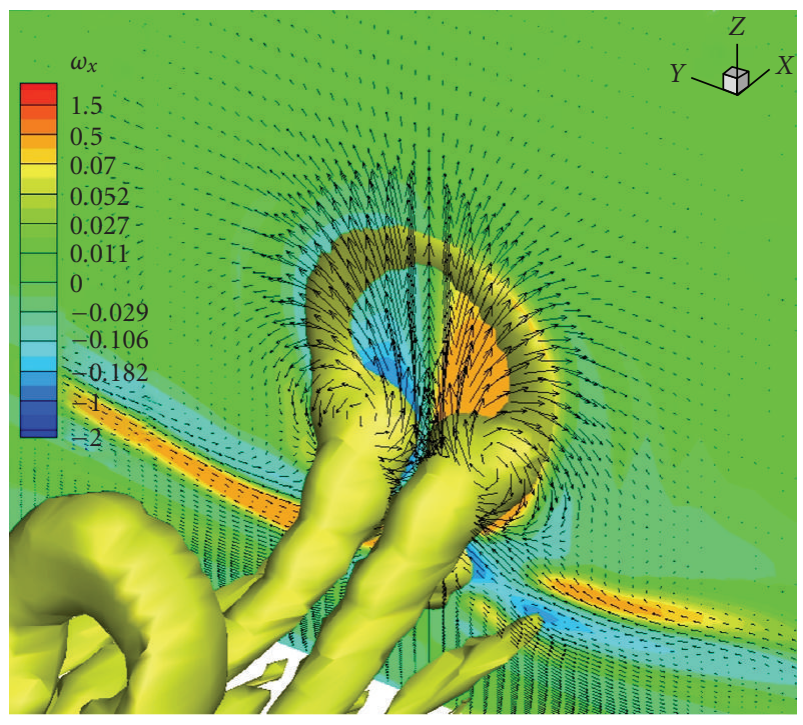

(a)

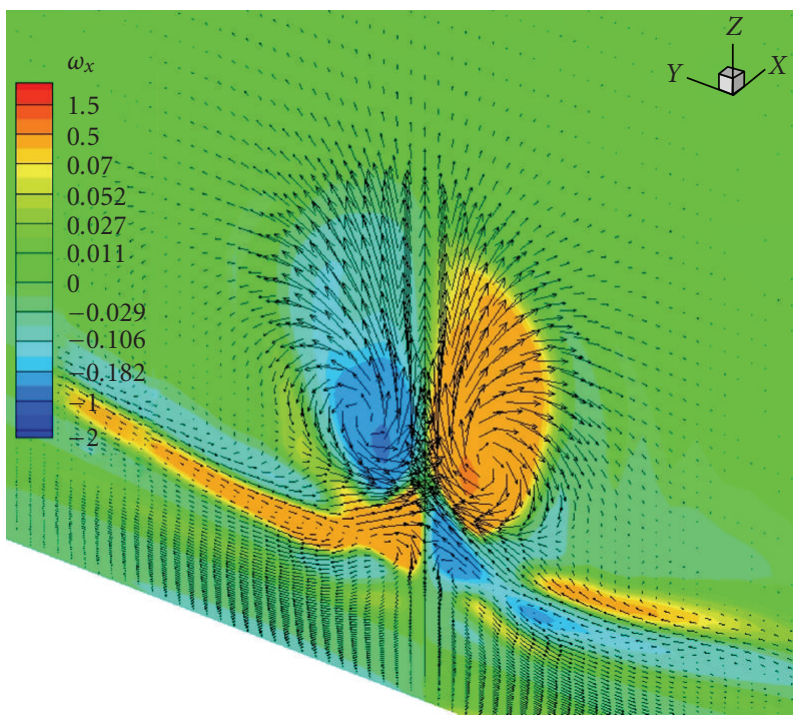

(b)

FIgURE 10: Formation process of ring-like vortices.
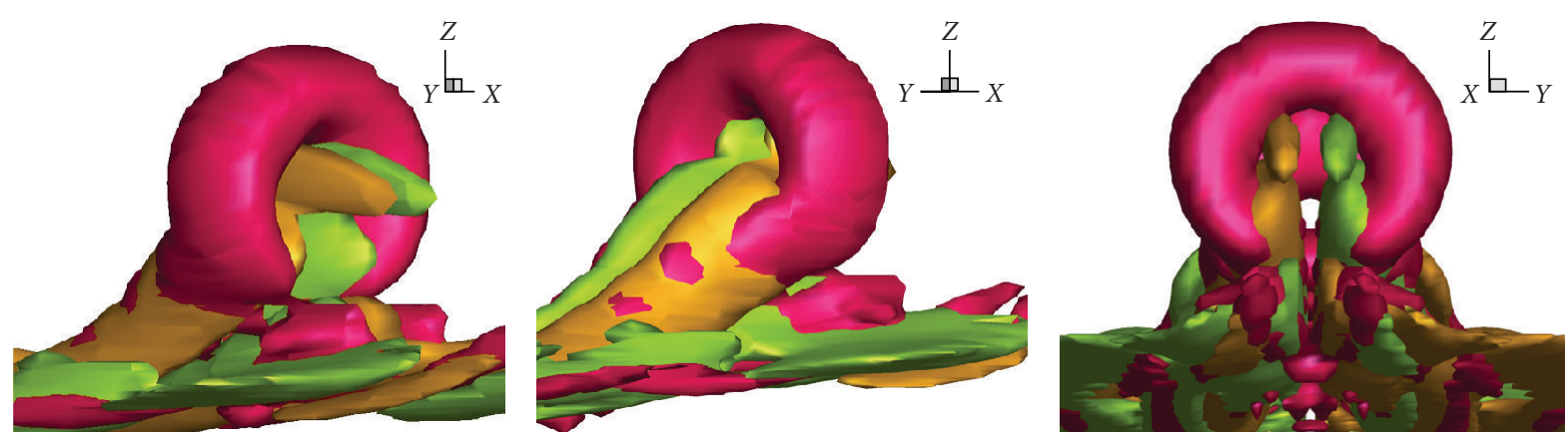

FIGURE 11: Four-vortex tube and ring-like vortices from three different view angles. Ring like vortex given by our DNS. 


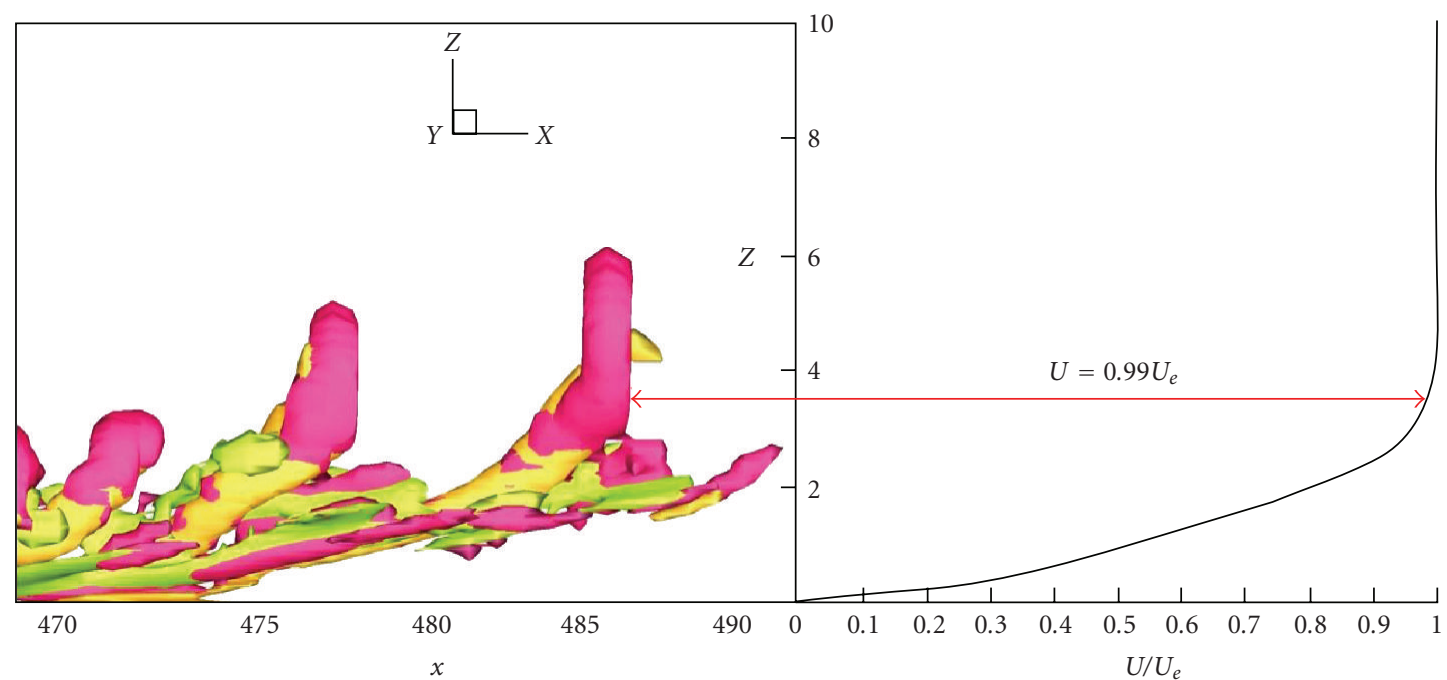

FIGURE 12: The position of ring-like vortices in boundary layer $\left(z=3.56, U=0.99 U_{e}\right)$

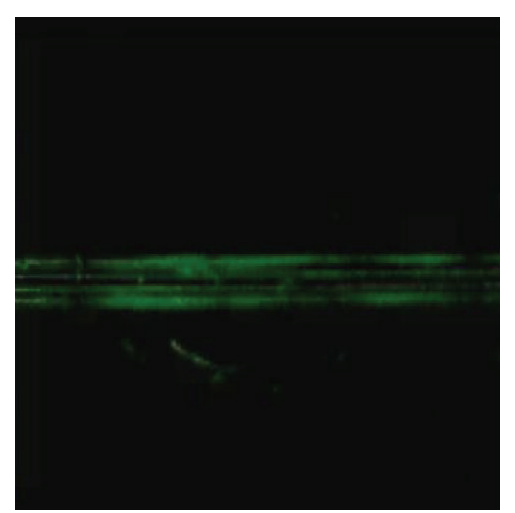

(a)

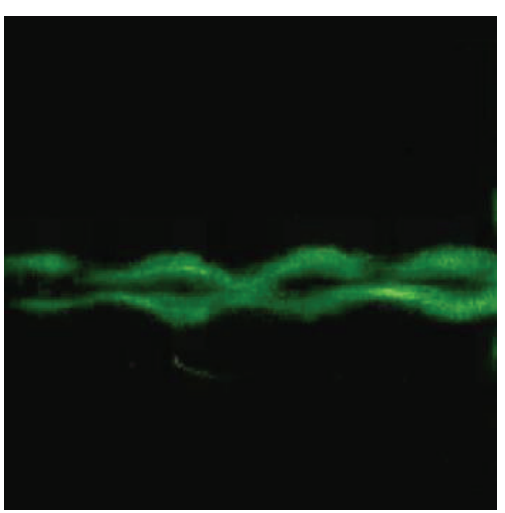

(b)

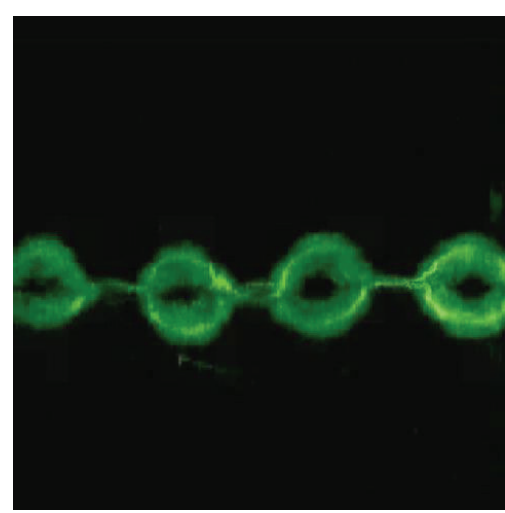

(c)

FIgURE 13: Crow theory about vortex ring chain formation (provided by Dr. Kachanov at FTT09 Short Course).

used instead of artificial dissipation. An implicit sixth-order compact scheme for space filtering is applied to the primitive variables $u, v, w, \rho, p$ after a specified number of time steps.

The governing equations are solved explicitly by a 3rdorder TVD Runge-Kutta scheme [15]:

$$
\begin{gathered}
Q^{(0)}=Q^{n}, \\
Q^{(1)}=Q^{(0)}+\Delta t R^{(0)}, \\
Q^{(2)}=\frac{3}{4} Q^{(0)}+\frac{1}{4} Q^{(1)}+\frac{1}{4} \Delta t R^{(1)}, \\
Q^{n+1}=\frac{1}{3} Q^{(0)}+\frac{2}{3} Q^{(2)}+\frac{2}{3} \Delta t R^{(2)},
\end{gathered}
$$

$C F L \leq 1$ is required to ensure the stability.

The adiabatic and the nonslipping conditions are enforced at the wall boundary on the flat plate. On the far field and the outflow boundaries, the nonreflecting boundary conditions [16] are applied.
The inflow is given in the form of

$$
q=q_{\mathrm{lam}}+A_{2 d} q_{2 d}^{\prime} e^{i\left(\alpha_{2 d} x-\omega t\right)}+A_{3 d} q_{3 d}^{\prime} e^{i\left(\alpha_{3 d} x \pm \beta y-\omega t\right)},
$$

where $q$ represents $u, v, w, p$, and $T$, while $q_{\text {lam }}$ is the Blasius solution for a two-dimensional laminar flat plate boundary layer. The streamwise wavenumber, spanwise wavenumber, frequency, and amplitude are

$$
\begin{aligned}
\alpha_{2 d} & =0.29919-i 5.09586 \times 10^{-3}, & & \beta= \pm 0.5712, \\
\omega & =0.114027, & A_{2 d}=0.03, & A_{3 d}=0.01,
\end{aligned}
$$

respectively. The T-S wave parameters are obtained by solving the compressible boundary layer stability equations [17].

The computational domain is displayed in Figure 1. The grid level is $1920 \times 128 \times 241$, representing the number of grids in streamwise $(x)$, spanwise $(y)$, and wall normal $(z)$ directions. The grid is stretched in the normal direction and uniform in the streamwise and spanwise directions. The length of the first grid interval in the normal direction at the entrance is found to be 0.43 in wall unit $\left(Y^{+}=0.43\right)$. 

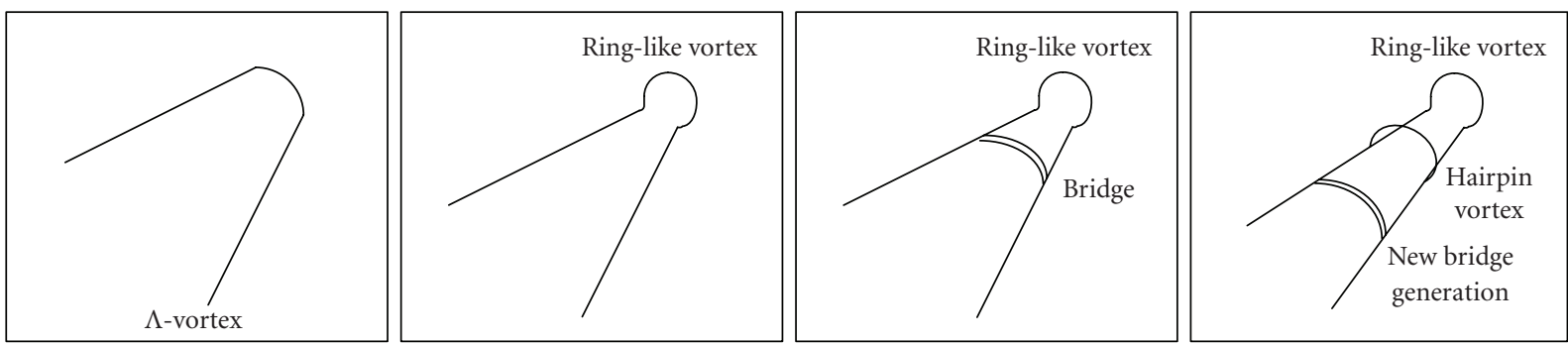

(a)

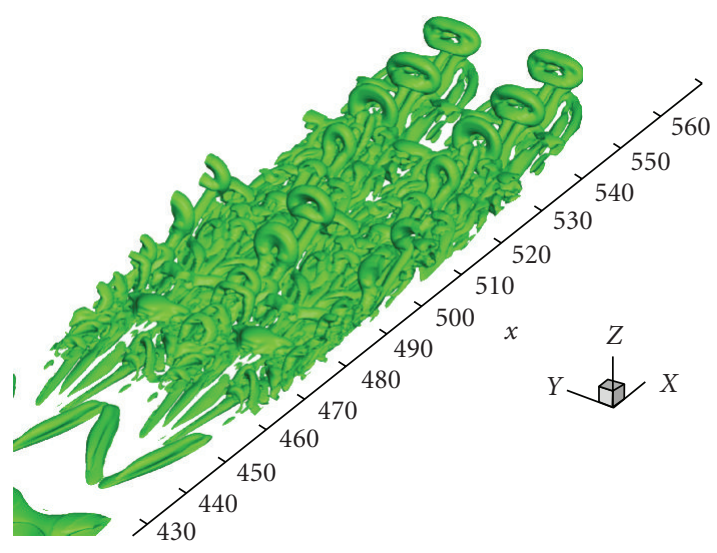

(b)

FIGURE 14: (a) Sketch for mechanism of multi-ring generation. (b) Multiple ring formation.

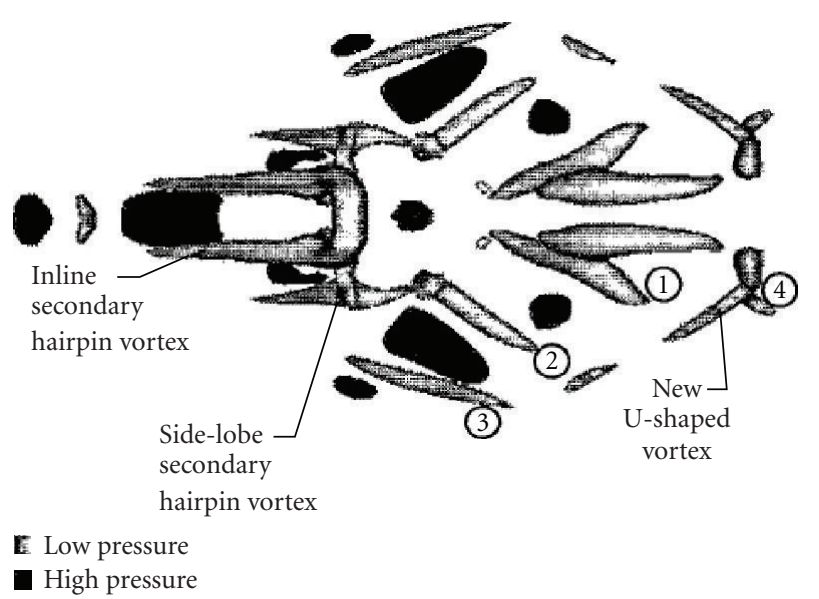

Figure 15: U-shaped vortex [9].

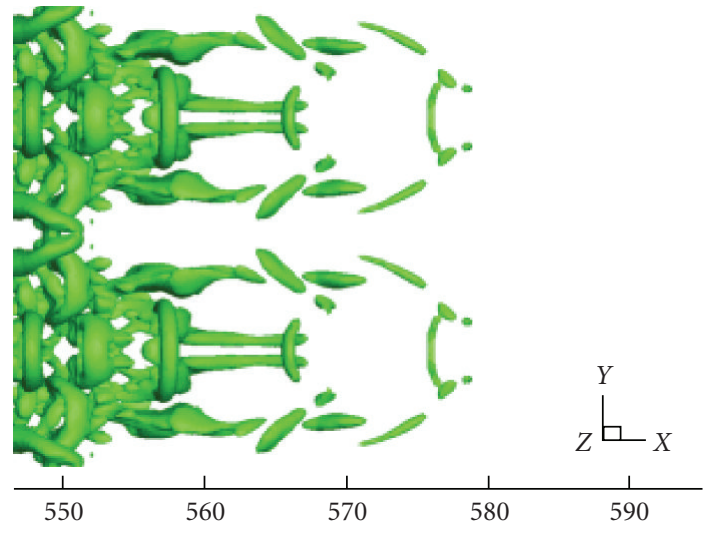

Figure 16: Our new DNS with a different $\lambda$-value.

The parallel computation is accomplished through the Message Passing Interface (MPI) together with domain decomposition in the streamwise direction. The computational domain is partitioned into $N$ equally sized subdomains along the streamwise direction (Figures 1 and 2). $N$ is the number of processors used in the parallel computation. The flow parameters, including Mach number, Reynolds number are listed in Table 1.

\section{Verification and Validation}

The skin friction coefficient calculated for the time- and spanwise-averaged profile is displayed in Figure 3. The spatial evolution of skin friction coefficients of laminar flow is also plotted out for comparison. It is observed from these figures that the sharp growth of the skin-friction coefficient occurs after $x \approx 450 \delta_{\text {in }}$, which is defined as the "onset point". The skin friction coefficient after transition is in good agreement with the flat-plate theory of turbulent boundary layer by Cousteix in 1989 [18]. 


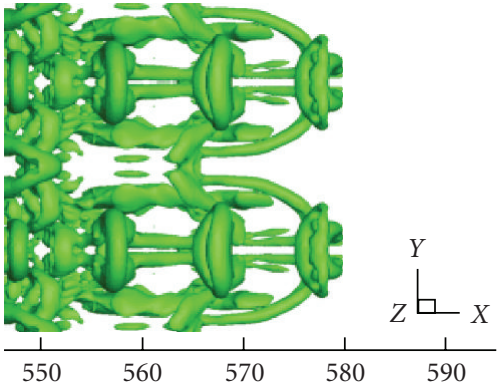

(a) Top view

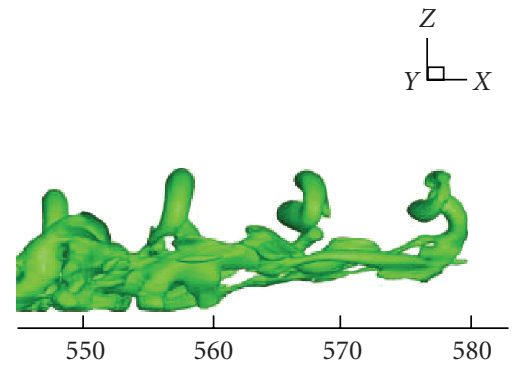

(b) Side view

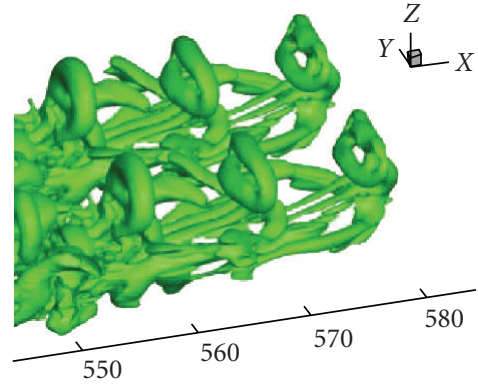

(c) Angle view

FIGURE 17: View of young turbulence spot head from different directions $(t=8.8 T)$.

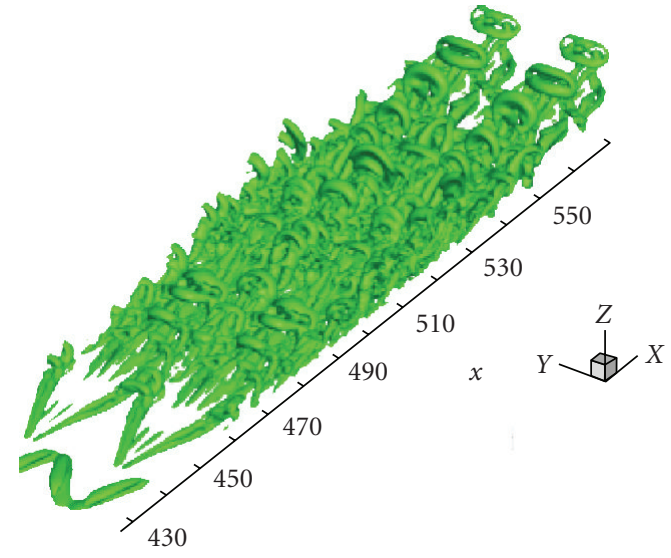

(a) Angle view

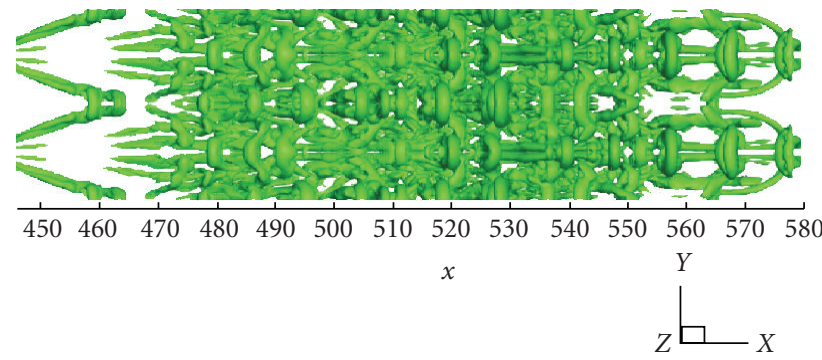

(b) Top view

FIgURE 18: 3D angle view and $2 \mathrm{D}$ top view of the young turbulence spot $(t=8.8 T)$.

Time- and spanwise- averaged streamwise velocity profiles for various streamwise locations in two different grid levels are shown in Figure 4 . The inflow velocity profiles at $x=300.79 \delta_{\text {in }}$ is a typical laminar flow velocity profile. At $x=$ $632.33 \delta_{\text {in }}$, the mean velocity profile approaches a turbulent flow velocity profile (Log law). Note that there are quite few publications (e.g., Figure 4(c)) which have large discrepancy in velocity profile with Log law.

A vortex identification method introduced by Jeong and Hussain [12] is applied to visualize the vortex structures by using an iso-surface of a $\lambda_{2}$-eigenvalue. The vortex cores are found by the location of the inflection points of the pressure in a plane perpendicular to the vortex tube axis. The pressure inflection points surround the pressure minimum that occurs in the vicinity of the vortex core. By this visualization method, the vortex structures shaped by the nonlinear evolution of T-S waves in the transition process are shown in Figure 5(A). The evolution details are studied in our previous papers [19-23], and the formation of ring-like vortices chains is consistent with the experimental work (see [13], Figure 5(B)) and previous numerical simulation by Bake et al. [3].

\section{New Discoveries and Mechanisms Revealed by Our DNS}

4.1. Mechanism on Secondary Streamwise Vortex Formation. As shown in Figure 6(a), the primary vortex induces a backward velocity which generates negative vorticity by the wall surface due to the zero velocity on the wall surface. However, negative vorticity generation does not mean formation of the secondary vortex tube. The secondary vortex tube must be generated by the flow separation. This means that the flow direction near the wall must change the sign from backward to forward (Figure 6(b)). This process is directly shown by the DNS results in Figures 7(a) and 7(b). The separation can only be done by pressure gradients. The question is where the pressure gradients come from. From Figure 8, we can clearly find that the streamwise vortex center is the low pressure center and the pressure outside the primary vortex tube is much higher than the center of the primary vortex center. Also from Figure 8, it is shown that the prime vortex changes the streamwise pressure gradient. These pressure gradients change the flow direction from backward to forward, and the secondary vortex tube forms, separates from the wall surface, and rises up. 


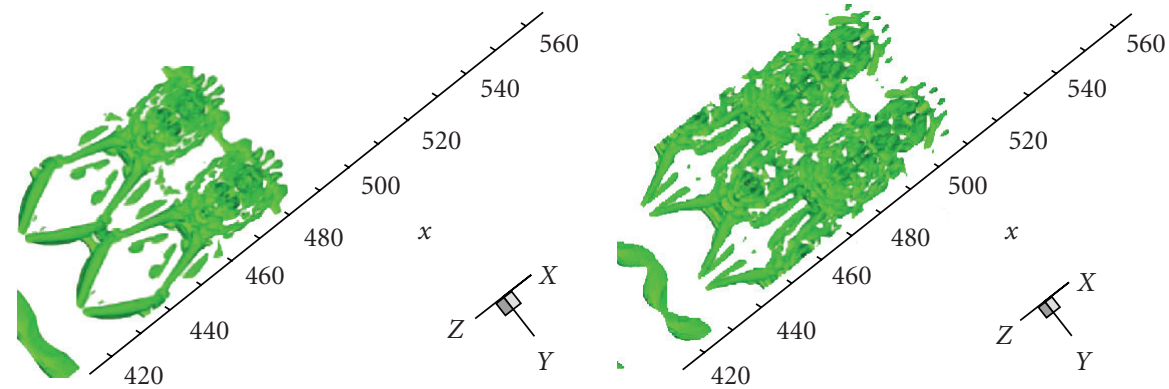

(a) $t=7.0 \mathrm{~T}$

(b) $t=7.5 T$

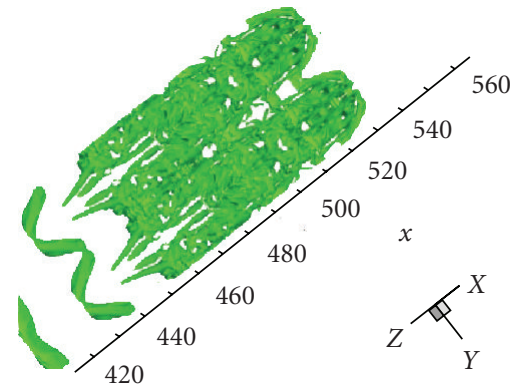

(c) $t=8.0 T$

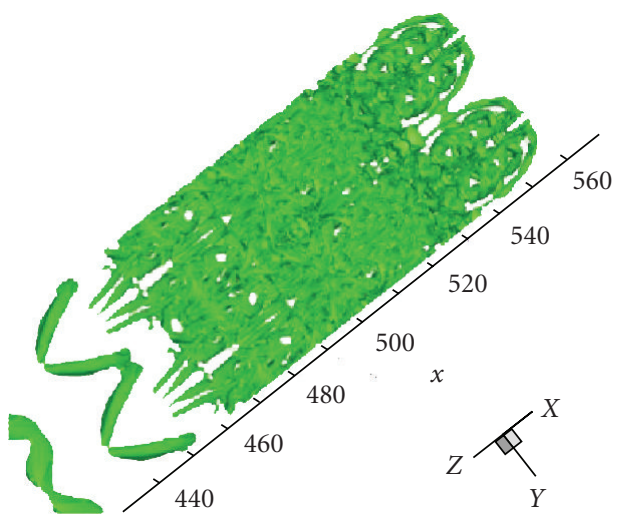

(d) $t=8.5$

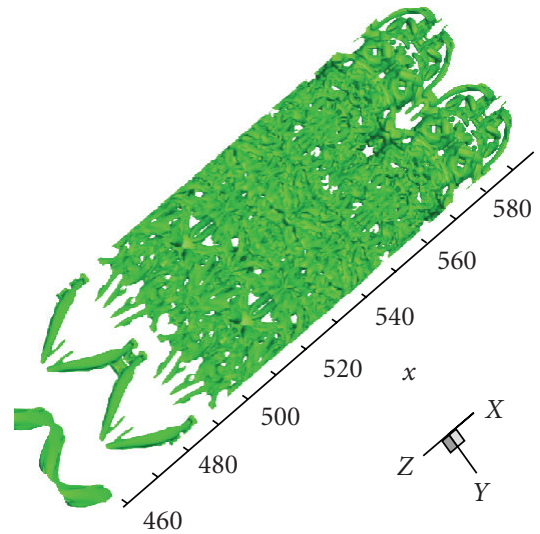

(e) $t=8.8 T$

Figure 19: Small length scale vortex generation at different time steps (view up from bottom): small length scale vortices are generated by the solid wall near the ring necks from the beginning to the end.

4.2. Mechanism of First Ring Formation. Ring-like vortices play a vital role in the transition process of wall boundary layer flow. Based on our numerical simulation, the generation of ring-like vortices is the product of the interaction between the prime streamwise vortices and secondary streamwise vortices. A ring is the consequence when the interaction between prime and secondary streamwise vortex becomes strong enough. The formation details are shown in Figure 9. The prime streamwise vortices are inside the ring, while the secondary streamwise vortices are outside the ring. According to the velocity vector trace, the prime streamwise vortices push flow outward, and the secondary streamwise vortices pull flow in from underneath (Figure 10). These movements bend and stretch the hairpin vortices and then generate a narrow neck. Finally a ring is formed at the tip of the $\Lambda$-vortices. For comparison, the ring-like vortex shape given by Moin et al. [7] is taken as a reference. The detail structure of four vortex tubes and ring-like vortices is depicted in Figure 11 from three different view angles. They are quite different.

Apparently, our new DNS results show that the ring is a perfect circle (Figure 11(b)) not a deformed shape as shown by Moin et al. [7], the ring stands perpendicularly with 90 degrees not 45 degrees as shown by Moin et al. [7], and, more important, "ring pinch off" given by Moin et al. [7] is never found. The ring-like vortex has its own coherent structure as we show in Figure 11.

The profile in Figure 12 is a spanwise and time average streamwise velocity profile at location $x=486$ (the location of first ring-like vortices). As shown in Figure 12, the ringlike vortices are located outside the boundary layer or in other words located in the inviscid zone $(z=3.56, U=$ $\left.0.99 U_{e}\right)$. Because the leading rings are in the inviscid zone where the flow is almost uniform and isotropic, the shape of the ring is almost perfectly circular. It is believed that the velocity in normal direction is almost zero in the inviscid zone and the ring stands almost perpendicularly.

4.3. Mechanism of Multiple Ring Formation. Crow theory [8] has been considered as the mechanism of multiple ring formation [2]. Figure 13 shows a typical vortex chain formation by Crow theory. However, there is no evidence to prove such a mechanism for flow transition. According to our DNS, Figure 14(a) gives a schematic about the process of a single ring and multiple ring formation. Following the first Helmholtz vorticity conservation law, when the vortex tube is stretched and rolls up, the tube is narrowed, and an additional bridge is developed across the two legs of the 


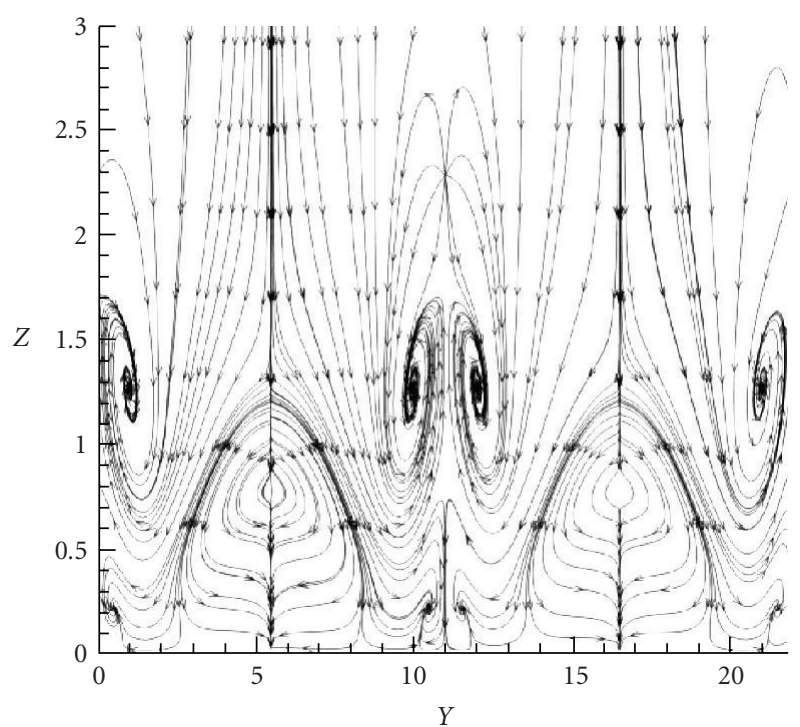

(a) $t=7.0 T$ and $x=442 \delta_{\text {in }}$. Primary $z=1.3 \delta_{\text {in }}$, Secondary $z=0.8 \delta_{\text {in }}$, Third $z=0.2 \delta_{\text {in }}$

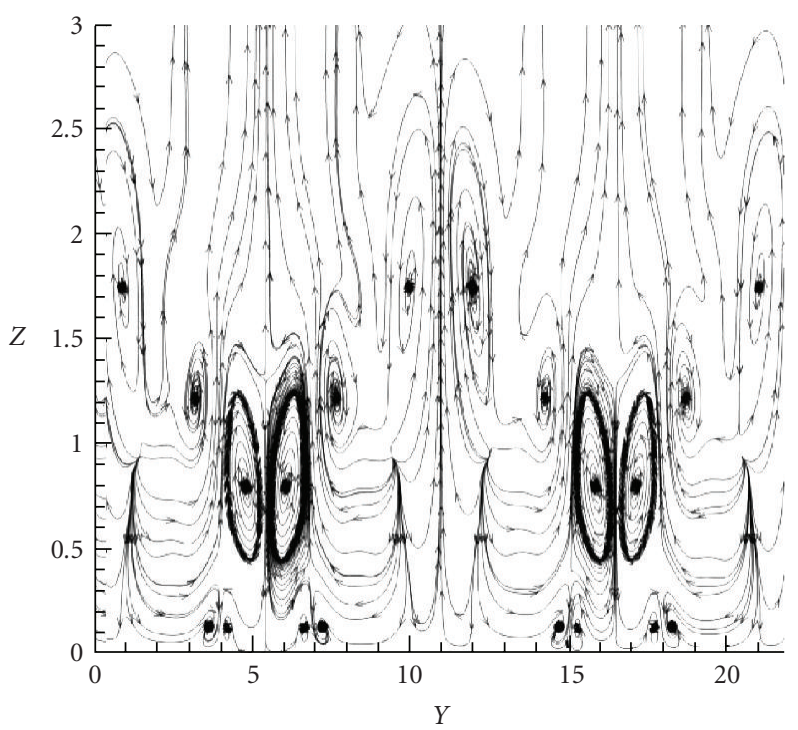

(b) $t=7.8 \mathrm{~T}$ and $x=475 \delta_{\text {in }}$. Induced small vortex at $z=0.2 \delta_{\text {in }}$

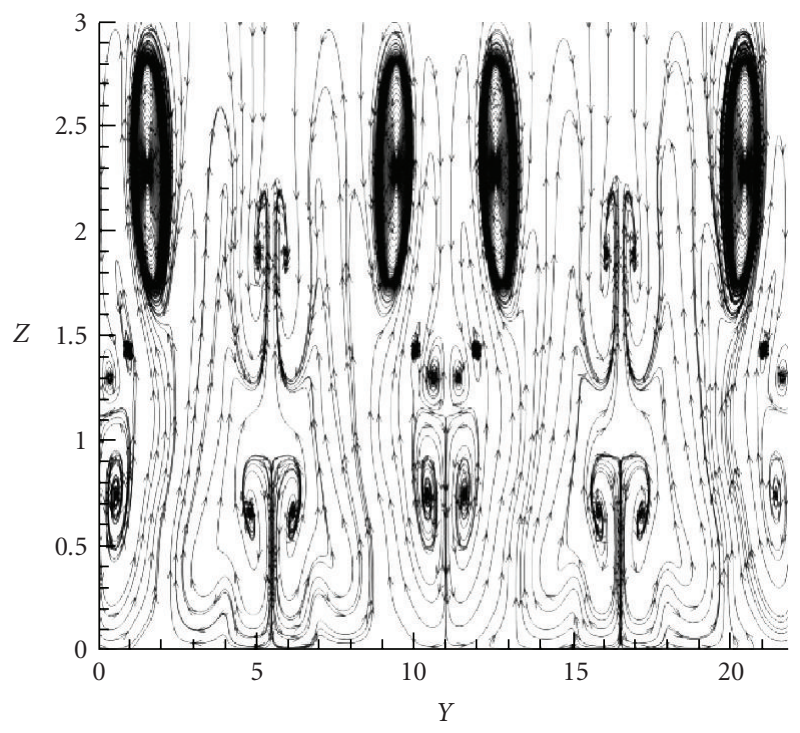

(c) $t=8.2 T$ and $x=515 \delta_{\text {in }}$. More small vortices are generated

FIGURE 20: Small vortices are generated by the wall surface at different time steps and streamwise locations by interaction with secondary vortices and positive spikes.

prime vortex tube to maintain the vorticity conservation. Consequently, the second, third, and so forth. rings are formed. Figure 14(b) shows that the multiple rings (over 8 rings) are formed and the first heading ring (right) is skewed and sloped (not perpendicular any more). From our new DNS, the so-called "spike" is nothing but formation of multiring (bridge) vortex structure which is quite stable. The multiple rings were generated one by one but not simultaneously like Crow theory, and the rings are perpendicular to the wall not parallel to the wall like Crow theory. Apparently, we cannot find any links between the Crow theory and the multiple ring formation.
4.4. U-Shaped Vortex Development. A description of the development of the U-shaped vortex can be found in the paper by Singer and Joslin [9]. Figure 15 has a 2D top view and uses low pressure contour to represent the vortex tube, which could lead to a misinterpretation that the secondary vortex is broken in to smaller pieces. Figure 16 is a $2 \mathrm{D}$ top view given by our new DNS with a different $\lambda_{2}$-value which gives a similar structure to Figure 15. However, there is no evidence that the secondary vortex breaks down. Let us look at the head of the so-called "turbulence spot" from different directions of view (Figure 17). The large vortex structure of the leading head of the turbulence spot is stable and is 


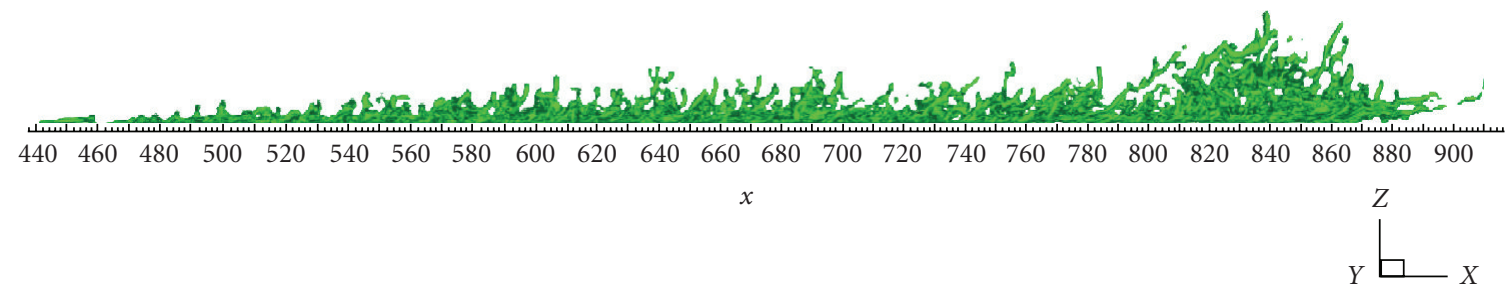

(a) Side view

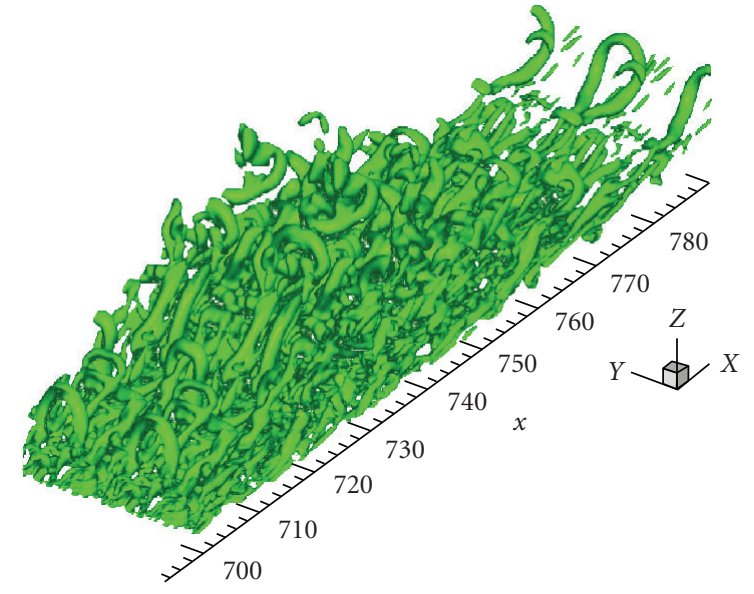

(b) Angle view (locally enlarged)

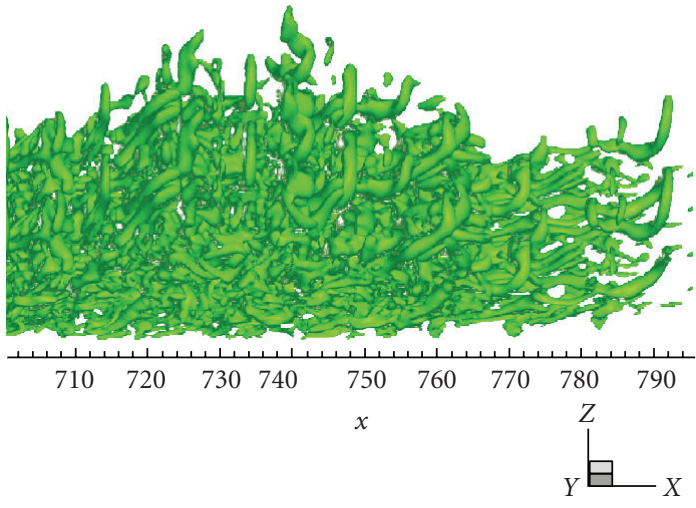

(c) Side view (locally enlarged)

FIGURE 21: Vortex cycle overlapping and boundary layer thickening.

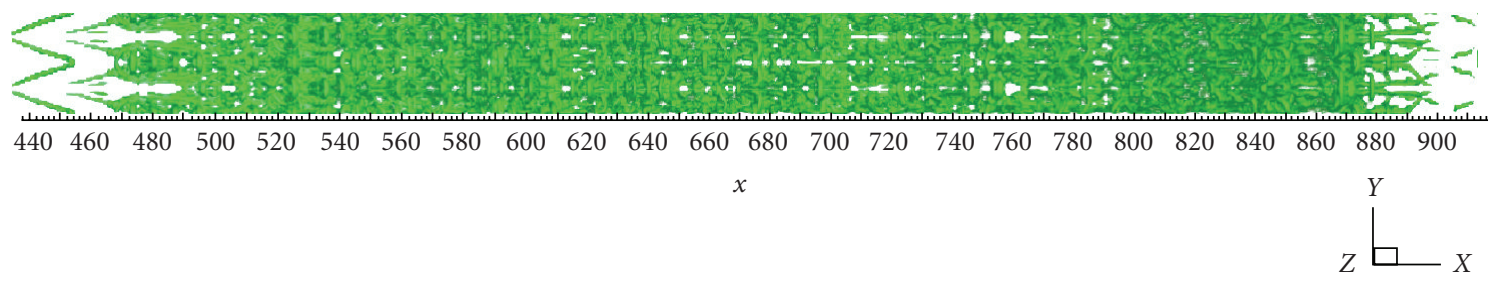

(a) Top view of ring cycle overlapping

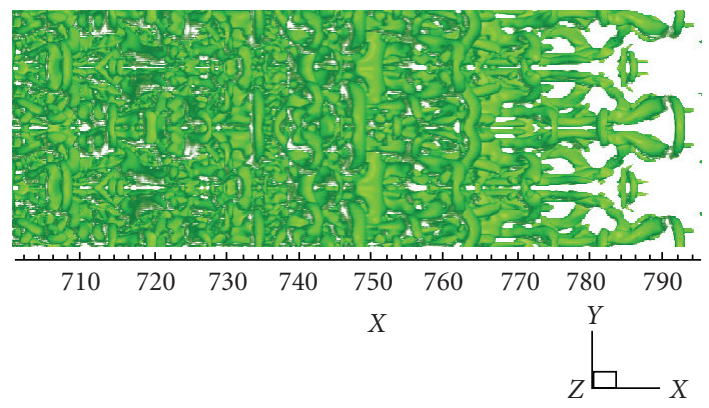

(b) Top view (locally enlarged)

Figure 22: Ring cycle overlapping, but never mixing.

still there. It travels for a long distance and never breaks down. We found that the head shape looks U-shaped, but the basic vortex structure really does not change and the heading hairpin vortex never breaks down. The real situation is that the heading primary ring is still there but skewed and sloped, which leads to a disappearance of the second sweep.
The consequences are that no more energy is transported down to the boundary sublayer by the second sweep which is weakened in the area near the head of the spot. The small length-scale-structures located near the laminar bottom damped, and the originally existing $U$-shaped vortex is rising and becomes clearly. It was thought [9] that the U-shaped 


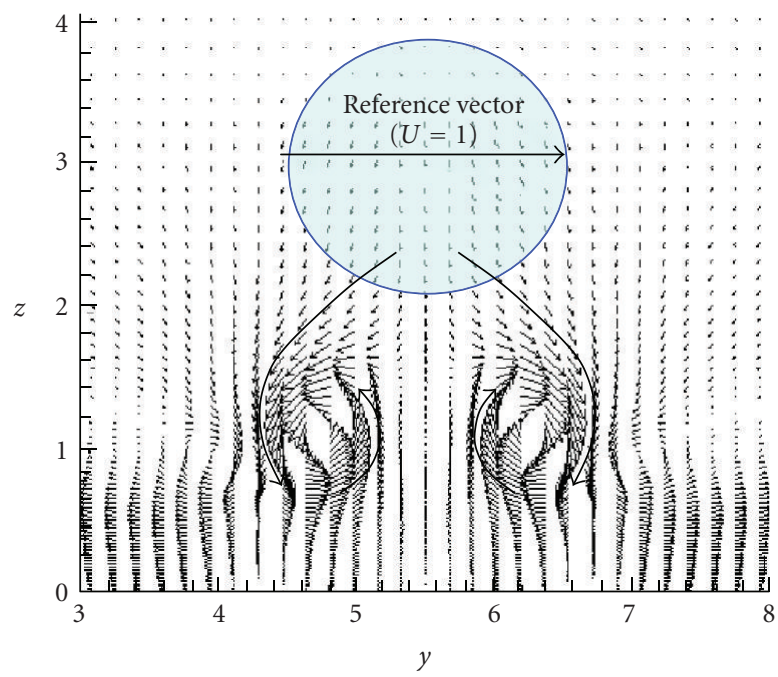

(a) Vector field

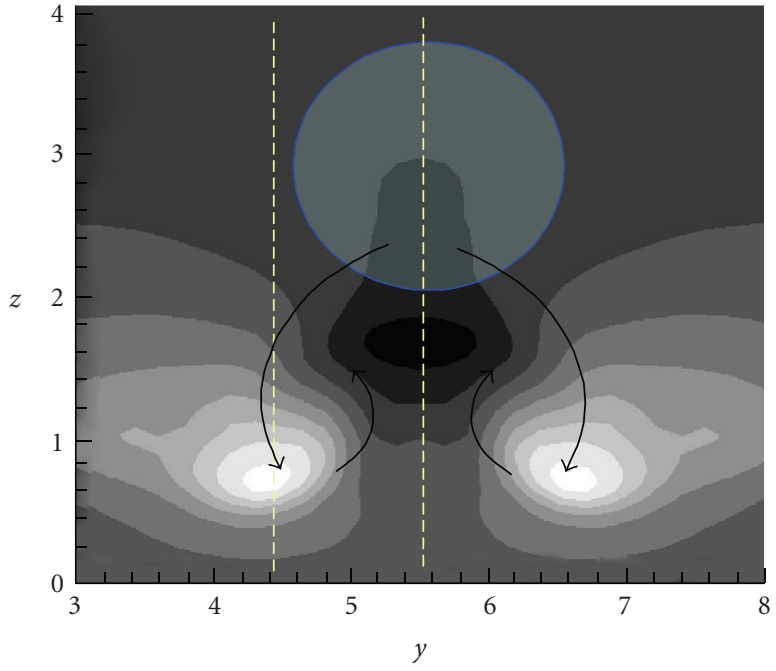

(b) Ten contour levels $-0.125 \sim 0.323$

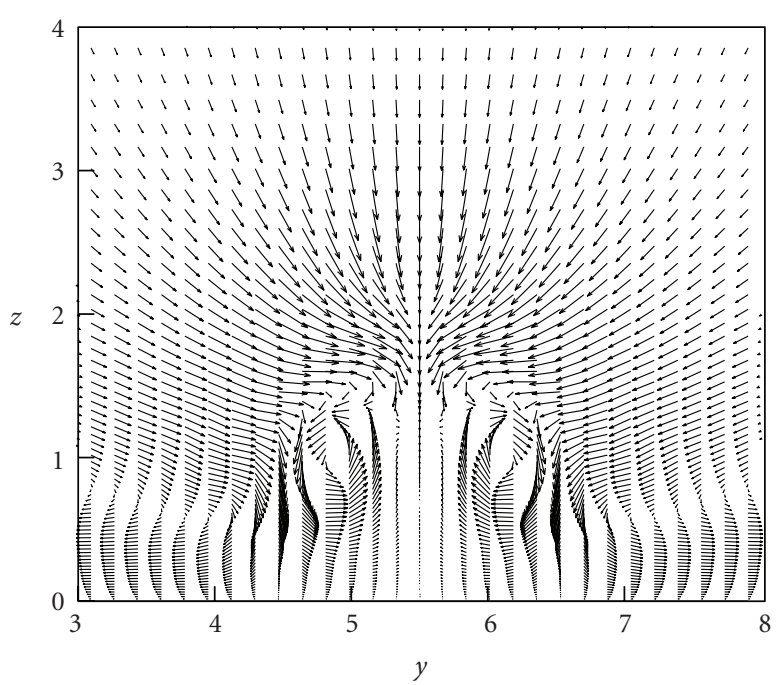

(c) Vector field at $x=462$

FIGURE 23: Vector field of $(v, w)$-velocity disturbance (a) and contours of streamwise velocity disturbance $u$ (b) in the $(y, z)$-plane at $t=6.6 T$, $x=464$, (c) $t=6.6 T$ and $x=462$. Thick arrows display downward and upward motions associated with sweep 2 and ejection 2 events, respectively.

vortex is a newly formed secondary vortex. However it really exists for some time. The reason that the U-shaped vortex is not clearly visible at previous times is that the $\mathrm{U}$-shaped vortex is surrounded by many small length scale structures. When the heading primary ring is perpendicular, it will generate a strong second sweep which brings a lot of energy from the inviscid area to the bottom of the boundary layer and makes that area very active. However, when the heading primary ring is no longer perpendicular, skewed and sloped, the second sweep disappears. The small length scale structures rapidly damp and the originally existing U-shaped vortex becomes clear since they are part of the large scale structure. Different from Singer and Joslin [9], the U-shaped vortex is found not a secondary, but tertiary vortex with same sign of vorticity of the corresponding ring leg. Actually, the
U-shaped vortex is a second neck to supply vorticity to the ring (Figures 17(b) and 17(c)).

Due to the increase of the ring (bridge) number and vorticity conservation, the leading rings will become weaker and weaker until they cannot be detected, but they never break down (see Figure 17). The multiring structure is pretty stable and the rings can travel for long distances (Figure 18) because they are located in an inviscid area and the U-shaped neck provides more vorticity.

4.5. Mechanism of Small Length Scale Generation. It is natural that one question will be raised. The question is where the small vortex length scales come from? We believe that these small length scale vortices are generated by the interaction of secondary and third level vortices with the wall surface. 


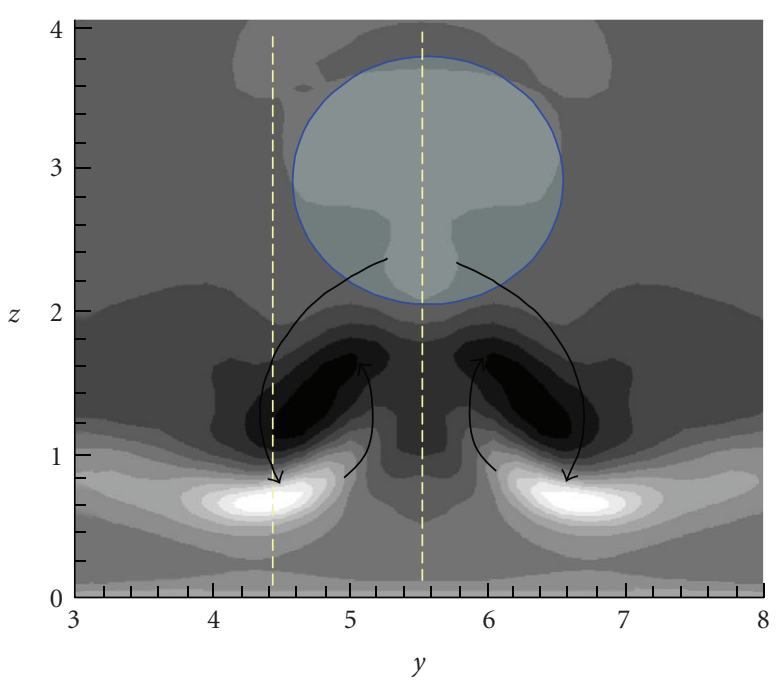

(a) $x=465.10$ contour levels $-0.456 \sim 0.718$

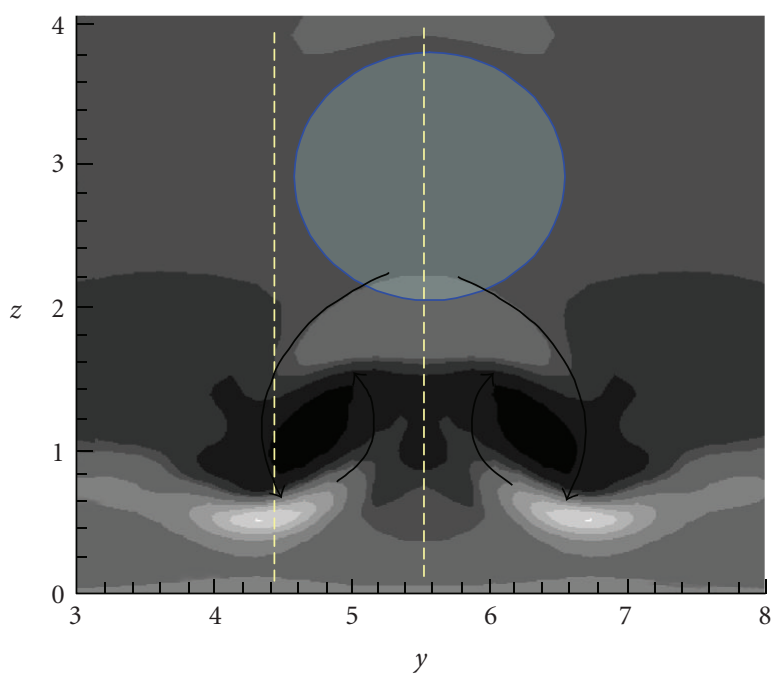

(b) $x=464.10$ contour levels $-0.698 \sim 1.202$

FIGURE 24: Contours of spanwise component of the vorticity disturbance in the $(y, z)$-plane at $t=6.6 T$. Light shades of gray correspond to high values.

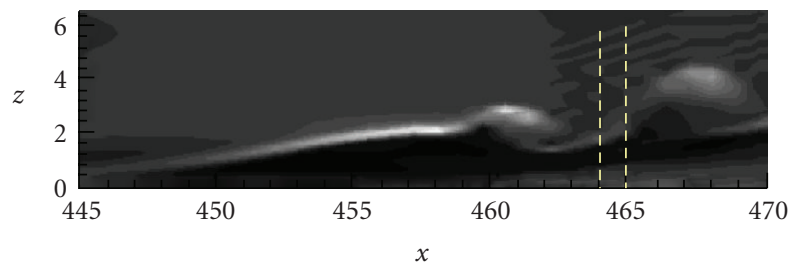

(a) $y=5.5 .10$ contour levels $-0.364 \sim 1.531$

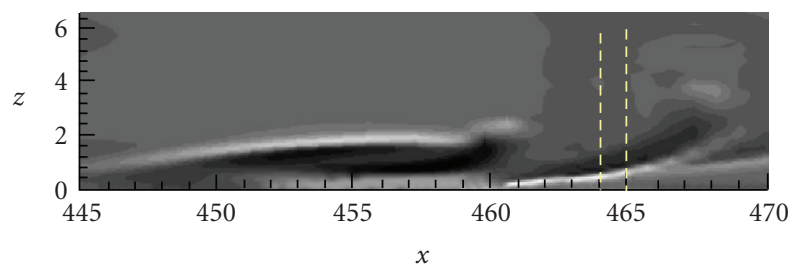

(b) $y=4.4 .10$ contour levels $-0.698 \sim 1.202$

Figure 25: Contours of spanwise component of the vorticity disturbance in the $(x, z)$-plane at $t=6.6 T$. Light shades of gray correspond to high values.

As we know, the vorticity can only be given or generated in boundaries, but it cannot be generated inside the flow field. Actually, the wall surface is the sole source of the vorticity generation for boundary later. Our new DNS gives the preliminary answer that the small length scale vortices are generated by the wall surface and near the wall surface as they are generated by second sweep instead of hairpin vortex breakdown. Actually, vortex breakdown is theoretically impossible and practically never found. The animations of our DNS results give details of flow transition at every stage and new mechanisms of small length scale vortex generation. We provide some snap shots here (Figure 19). Since we believe that the small vortices are generated by the wall surface and near wall region, we take snap shots in the direction of view from the bottom to top. It is easily found that the small length scale structures first appear on the wall near the ring neck area in the streamwise direction.

All evidences provided by our new DNS confirm that the small length scale vortices are generated near the bottom (Figures 19 and 20) by secondary vortices or third level vortices, especially by the positive spike caused by strong second sweeps which bring high streamwise velocity from inviscid area to sublayer.
4.6. Vortex Cycle Overlapping. As we discussed before, the ring head is located in the inviscid area and has much higher moving speed than the ring legs which are located near the bottom of the boundary layer. It is the reason that the hairpin vortex is stretched and multiple rings are generated. This will lead to an overlapping of second ring cycle upside of the first ring cycle. However, no mixing of two cycles is observed by our new DNS (Figures 21 and 22). The second ring is separated from first ring cycle by a secondary group of rings which are generated by the wall surface, separated from wall, and convected to downstream. This is the reason why the transitional boundary layer becomes thicker and thicker.

4.7. High Shear Distribution. The $(v, w)$-velocity disturbance vectors and the $u$-velocity contours are presented in Figure 23 in the $(y, z)$-plane. The projection of the approximate location of the first vortex ring onto this $(y, z)$ plane is indicated in Figure 23 with semitransparent circles. It is seen that there are two strong downdraft jets produced by the first vortex ring behind it at two sides of the structure centerline. The existence of these jets was called "sweep 2" events $[5,24]$. 


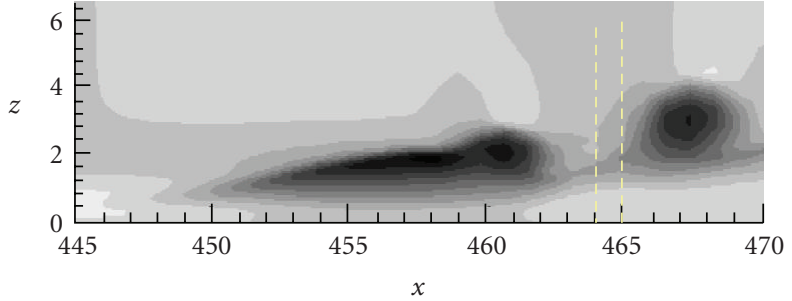

(a) $y=5.5$. ten contour levels $-0.474 \sim 0.150$

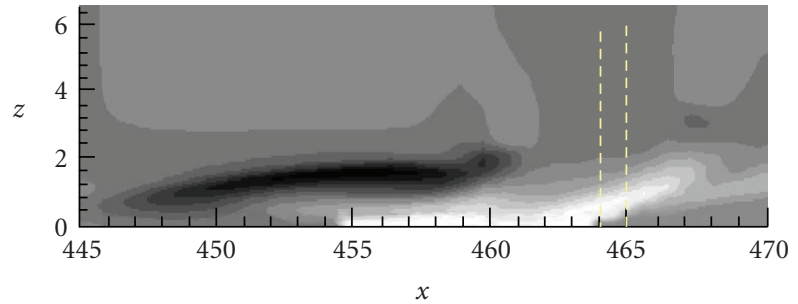

(b) $y=4.4$. ten contour levels $-0.332 \sim 0.340$

FIGURE 26: Contours of streamwise velocity disturbance $u$ in the $(x, z)$-plane at $t=6.6 T$. Light shades of gray correspond to high values.

Formation of a low speed region between the $\Lambda$-vortex legs and appearance of an HS-layer slightly above and inside of the $\Lambda$-vortex can be found in Figures 24, 25, and 26. It is found (based on comparison of instantaneous fields calculated for various time instants) that the low-speed streaks and high-shear layers associated with the ejection 2 events seem to become weaker when the ring-like vortex propagates downstream and finally disappears. This result is consistent with observations by Bake et al. [3]. They believe that low-speed streaks and high-shear layers become weak and finally disappear because the "tail vortices" connecting the ring- like vortices move towards the wall. Based on our new DNS results, it shows that the main reason for the above phenomena is that in the middle between the two positive spikes near the ring neck, there is an area where the normal velocity component is almost zero (see Figures 23(a) and $23(c))$.

\section{Conclusions}

Based on our new DNS study, the following conclusions can be made.

(1) Vorticity is generated by the wall. The secondary vortex can be developed only through flow separation which is caused by the adverse pressure gradient.

(2) A vortex tube inside the flow field cannot have ends, and thus vortex ring is the only form existing inside the flow field because the ring has no ends, head, or tail.

(3) There are four streamwise vorticity lines around the $\Lambda$-shaped vortex, two primary inside and two secondary outside. The inside two primary streamwise vorticity lines pull out the $\Lambda$-shaped vortex to form the ring, but the outside two secondary vorticity lines push the $\Lambda$-shaped vortex to form the neck. Therefore, the formation of a ring-like vortex is the interaction between the primary and secondary streamwise vorticity lines.

(4) The leading ring-vortices are almost perfectly circular and stand almost perpendicularly. The so-called "ring pinch off" is not found.

(5) The stretched vortex tube will be narrowed with stronger vorticity along with faster rotation as a result of narrowing. According to the first Helmoholtz vorticity conservation law, this will lead to development of a bridge and further become a second ring, and so on.

(6) U-shaped vortex is a coherent structure and serves as second neck to supply the rings.

(7) Since the head of the hairpin vortex, that is, rings, is located in the inviscid area and the U-shaped vortex supplies vorticity to the ring, the consequent multiple ring-like vortex structure can travel for long distance in the flow field. The hairpin vortex will become weak until being nondetectable, but never breaks down.

(8) The small length scales are generated by the wall surface and near the wall region, but not by the hairpin vortex breakdown.

(9) The ring head speed is faster than the ring legs, which leads to ring cycle overlapping. Multiple ring cycle overlapping will lead to thickening of the transitional boundary layer. The multiple rings overlap, but never mix. They are separated by secondary rings which are generated by the wall surface, separated from the wall, and convected to downstream.

(10) The updraft motion leads to the $\Lambda$-shaped highspeed streaks and an HS-layer near the wall outside the $\Lambda$-vortex legs. However, the HS-layer disappears near the ring neck because the normal velocity in the near neck area is almost zero.

\section{Nomenclature}

$M_{\infty}$ : Mach number

Re: Reynolds number

$\delta_{\text {in }}:$ Inflow displacement thickness

$T_{w}$ : Wall temperature

$T_{\infty}$ : Free stream temperature

$L z_{\text {in }}$ : Height at inflow boundary

$L x$ : Length of computational domain along $x$ direction

$L y$ : Length of computational domain along $y$ direction

$x_{\text {in }}$ : Distance between leading edge of flat plate and upstream boundary of computational domain

$A_{2 d}$ : Amplitude of 2D inlet disturbance 
$A_{3 d}$ : Amplitude of 3D inlet disturbance

$\omega$ : Frequency of inlet disturbance

$\alpha_{2 d}$ : Streamwise wave number of inlet disturbance

$\beta$ : Spanwise wave number of inlet disturbance

$R: \quad$ Ideal gas constant

$\gamma$ : $\quad$ Ratio of specific heats

$\mu_{\infty}$ : Viscosity

$\omega_{x}$ : Streamwise vorticity

$\lambda_{2}$ : Const representing vortex tube surface.

\section{Acknowledgments}

This work was supported by AFOSR grant FA9550-08-10201 supervised by Dr. John Schmisseur. The authors are grateful to Texas Advanced Computing Center (TACC) for providing computation hours. This work is accomplished by using Code DNSUTA which was released by Dr. Chaoqun Liu at University of Texas at Arlington in 2009.

\section{References}

[1] L. Kleiser and T. A. Zang, "Numerical simulation of transition in wall-bounded shear flows," Annual Review of Fluid Mechanics, vol. 23, no. 1, pp. 495-537, 1991.

[2] V. I. Borodulin, V. R. Gaponenko, Y. S. Kachanov et al., "Latestage transitional boundary-layer structures. Direct numerical simulation and experiment," Theoretical and Computational Fluid Dynamics, vol. 15, no. 5, pp. 317-337, 2002.

[3] S. Bake, D. G. W. Meyer, and U. Rist, "Turbulence mechanism in Klebanoff transition: a quantitative comparison of experiment and direct numerical simulation," Journal of Fluid Mechanics, vol. 459, pp. 217-243, 2002.

[4] Y. S. Kachanov, "On a universal mechanism of turbulence production in wall shear flows," in Noteson Numerical Fluid Mechanics and Multidisciplinary Design, vol. 86 of Recent Results in Laminar-Turbulent Transition, pp. 1-12, Springer, Berlin, Germany, 2003.

[5] H. Guo, J. J. Wang, Q. X. Lian et al., "Spatial reconstruction of vortical structures in transitionalboundary layer based on synchronous hydrogen-bubble visualization," in Proceedings of the 12th International Conferenceon Methods of Aerophysical Research Part 1, pp. 118-124, Institute of Theoretical and Applied Mechanics, Novosibirsk, Russia, 2004.

[6] F. R. Hama and J. Nutant, "Detailed flow-field observations in the transition process in athick boundary layer," in Proceedings of the Heat Transfer and Fluid Mechanics Institute, pp. 77-93, Stanford University Press, Palo Alto, Calif, USA, 1963.

[7] P. Moin, A. Leonard, and J. Kim, "Evolution of a curved vortex filament into a vortex ring," Physics of Fluids, vol. 29, no. 4, pp. 955-963, 1986.

[8] S. C. Crow, "Stability theory for a pair of trailing vortices," AIAA Journal, vol. 8, no. 12, pp. 2173-2179, 1970.

[9] B. A. Singer and R. D. Joslin, "Metamorphosis of a hairpin vortex into a young turbulent spot," Physics of Fluids, vol. 6, no. 11 , pp. 3724-3736, 1994.

[10] C. B. Lee and J. Z. Wu, "Transition in wall-bounded flows," Applied Mechanics Reviews, vol. 61, no. 1-6, pp. 03080203080219, 2008.

[11] H. Schlichting and K. Gersten, Boundary Layer Theory, Springer, Berlin, Germany, 8th edition, 2000.

[12] . Jinhee Jeong and F. Hussain, "On the identification of a vortex," Journal of Fluid Mechanics, vol. 285, pp. 69-94, 1995.
[13] C. Lee and R. Li, "Dominant structure for turbulent production in a transitional boundary layer," Journal of Turbulence, vol. 8, no. 5, pp. 1-34, 2007.

[14] S. K. Lele, "Compact finite difference schemes with spectrallike resolution," Journal of Computational Physics, vol. 103, no. 1, pp. 16-42, 1992.

[15] C. W. Shu and S. Osher, "Efficient implementation of essentially non-oscillatory shock-capturing schemes," Journal of Computational Physics, vol. 77, no. 2, pp. 439-471, 1988.

[16] L. Jiang, H. Shan, and C. Liu, "Non-reflecting boundary conditions for DNS in curvilinear coordinates," in Proceedings of the 2nd AFOSR InternationalConference on DNS/LES, Recent Advances in DNS and LES, Rutgers-The State University of New Jersey, New Brunswick, NJ, USA, June 1999.

[17] M. R. Malik, "Numerical methods for hypersonic boundary layer stability," Journal of Computational Physics, vol. 86, no. 2, pp. 376-413, 1990.

[18] F. Ducros, P. Comte, and M. Lesieur, "Large-eddy simulation of transition to turbulence in a boundary layer developing spatially over a flat plate," Journal of Fluid Mechanics, vol. 326, pp. 1-36, 1996.

[19] L. Chen, D. B. Tang, X. B. Liu, M. Oliveira, and C. Q. Liu, "Evolution of the ring-like vortices and spike structure in transitional boundary layers," Science China: Physics, Mechanics and Astronomy, vol. 53, no. 3, pp. 514-520, 2010.

[20] L. Chen, X. Liu, M. Oliveira, and C. Liu, "DNS for ringlike vortices formation and roles in positive spikes formation," Tech. Rep. 2010-1471, AIAA, 2010.

[21] C. Liu and L. Chen, "DNS for late stage structure of flow transition on a flat-plate boundary layer," Tech. Rep. 20101470, AIAA, 2010.

[22] X.-B. Liu, Z.-Q. Chen, and C.-Q. Liu, "Late-stage vortical structures and eddy motions in a transitional boundary layer," Chinese Physics Letters, vol. 27, no. 2, Article ID 024706, 2010.

[23] X. Liu, L. Chen, and C. Liu, "Study of mechanism of ringlike vortex formation in late flow transition," Tech. Rep. 20101456, AIAA, 2010.

[24] H. Guo, Q. X. Lian, C. Pan et al., "Sweep and ejection events in transitional boundary layer. Synchronous visualization and spatial reconstruction," in Proceedings of the 13th International Conference on Methods of Aerophysical Research Part 4, pp. 192-197, Publishing House "Parallel", Novosibirsk, Russia, 2007. 

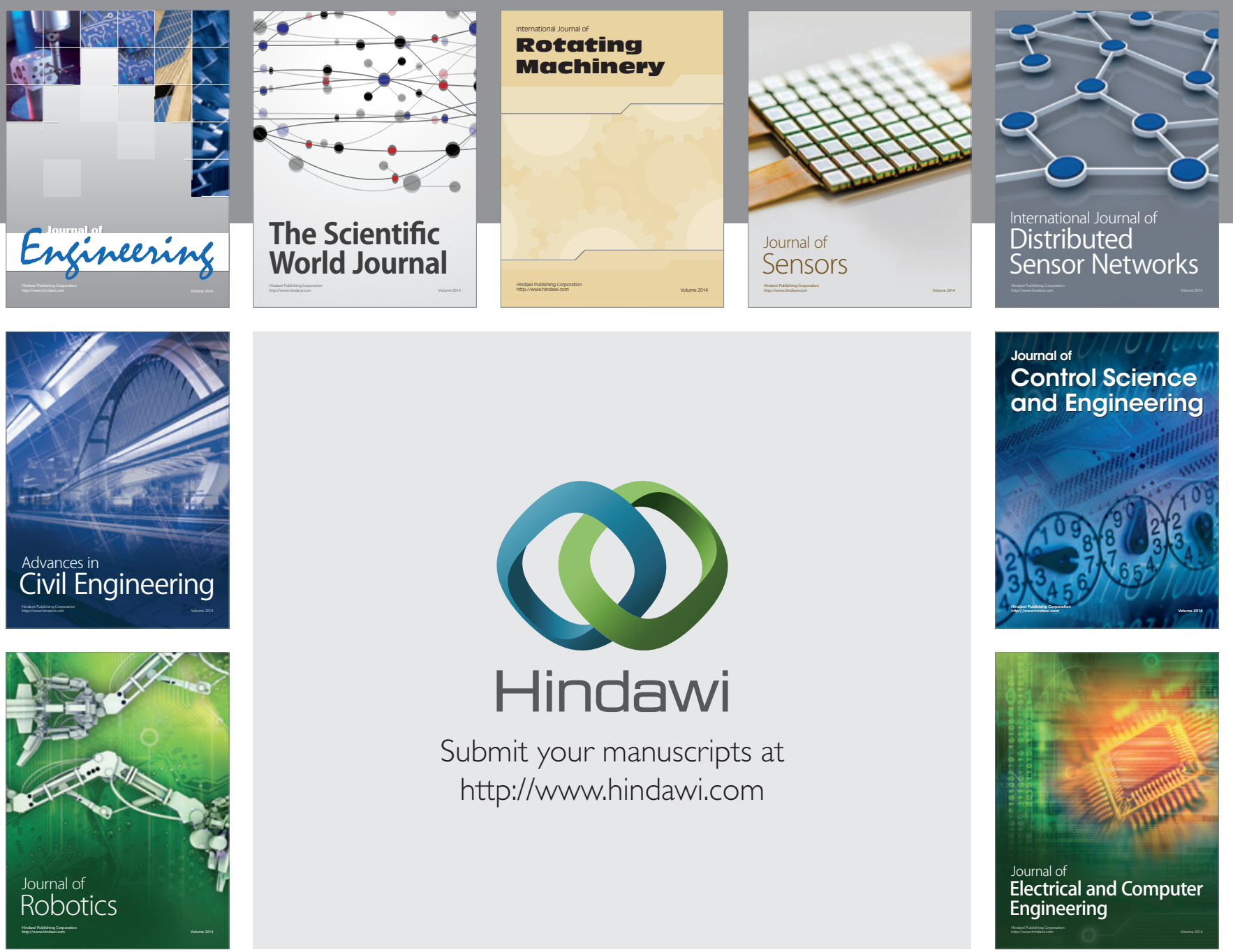

Submit your manuscripts at

http://www.hindawi.com
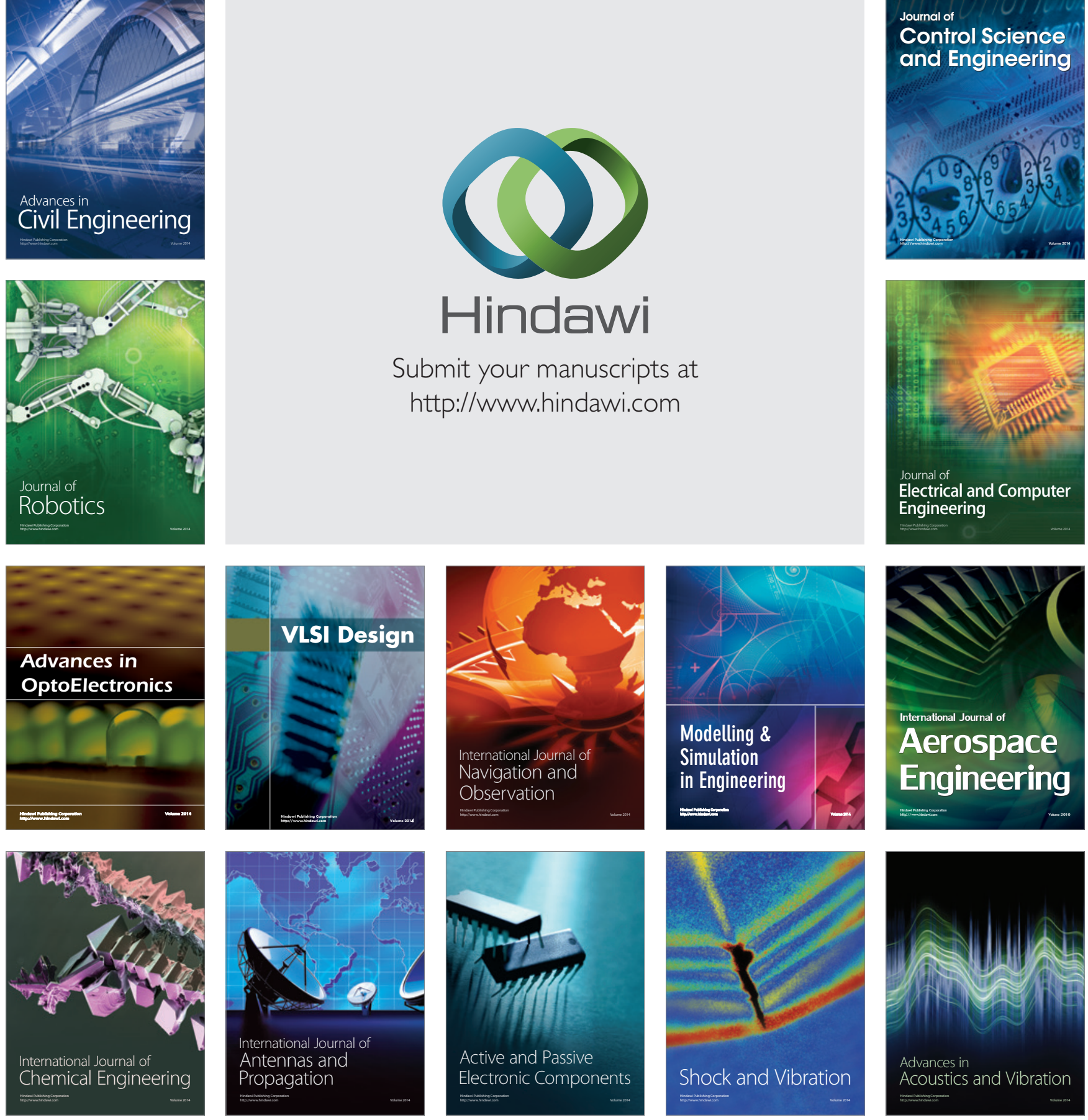